\title{
9. Menschliche Entwicklung und Menschenrechte
}

Michel Egger, Marie Thorndahl, Catherine Schümperli Younossian and Christoph Stamm

\section{OpenEdition}

\section{Journals}

Electronic version

URL: http://journals.openedition.org/sjep/220

DOI: $10.4000 /$ sjep.220

ISSN: 1663-9677

\section{Publisher}

Institut de hautes études internationales et du développement

\section{Printed version}

Date of publication: 1 avril 2004

Number of pages: 151-179

ISSN: 1660-5926

\section{Electronic reference}

Michel Egger, Marie Thorndahl, Catherine Schümperli Younossian und Christoph Stamm, « 9.

Menschliche Entwicklung und Menschenrechte », Schweizerisches Jahrbuch für Entwicklungspolitik [Online], 23-1 | 2004, Online erschienen am: 22 Juni 2010, abgerufen am 07 September 2020. URL http://journals.openedition.org/sjep/220 ; DOI : https://doi.org/10.4000/sjep.220 


\section{Menschliche Entwicklung und Menschenrechte*}

\subsection{Weltgipfel über die Informationsgesellschaft, Phase I, Genf, 10.-12. Dezember 2003}

Der Weltgipfel über die Informationsgesellschaft (World Summit on the Information Society, WSIS) gliedert sich in zwei Phasen. Die erste Phase wurde vom 10. bis zum 12. Dezember 2003 in Genf durchgeführt, die zweite Phase soll vom 16. bis zum 18. November 2005 in Tunis stattinden'. Der vorliegende Artikel zieht die Bilanz der ersten Phase und beleuchtet die wichtigsten noch umstrittenen Punkte, die im Jahr 2005 in Tunis auf der Tagesordnung stehen werden.

Bereits im Jahr 1998 unterbreitete Tunesien der Internationalen Fernmeldeunion (ITU) den Vorschlag, einen Kongress mit folgender Zielsetzung auszurichten: das Konzept der Informationsgesellschaft, in der die Telekommunikation eine zentrale Rolle spielt, unter Berücksichtigung der notwendigen und harmonischen Entwicklung der Regelungspolitik, der Netze und der Dienstleistungen zu konsolidieren ${ }^{2}$. Die Aufteilung in zwei Phasen - zurückzuführen auf das Unvermögen, sich auf eines der beiden Kandidatenländer festzulegen - beeinflusste den Verhandlungsprozess.

Die Verhandlungen der Genfer Phase drehten sich um zwei Dokumente, die von den Staaten zum Abschluss des Gipfels verabschiedet wurden. Die „Grundsatzerklärung " enthält eine gemeinsame Vision zur Informationsgesellschaft; der „Aktionsplan“ zielt darauf ab, die Informations- und Kommunikationstechnologien (IKT) in den Dienst der Entwicklung zu stellen und die „digitale Kluft“ zu überwinden.

Daneben verfolgt der Gipfel die Ambition, durch die Einsetzung von tripartiten Konsultationsmechanismen, welche die Staaten, Privatunternehmen und die Zivilgesellschaft in die Beschlussfassung einbinden, als Laboratorium der entstehenden neuen globalen Gouvernanz zu fungieren.

Unter den 11'000 Teilnehmern in Genf waren 176 Staaten, über 400 Organisationen der Zivilgesellschaft, rund hundert Privatunternehmen sowie über 600 Medien vertreten. Die Verfahrensregeln, welche die Teilnahmemodalitäten für

* Teil 9.1. von Michel Egger, Journalist und Koordinator für Entwicklungspolitik bei der Arbeitsgemeinschaft der Hilfswerke (Schweiz) und Marie Thorndahl, Sozial- und Wirtschaftswissenschaftlerin (Frankreich). Michel Egger war Gastredaktor und Marie Thorndahl Redaktionssekretärin für Band 2 des Schweizerischen Jahrbuchs für Entwicklungspolitik 2003, der das Dossier Informationsgesellschaft und internationale Zusammenarbeit. Development.com umfasst. Marie Thorndahl übernahm zudem die Koordination für die Herstellung der dem Band beigefügten CD-ROM.

Teil 9.2. von Catherine Schümperli Younossian, Forschungsbeauftragte am iuéd.

Teil 9.3. und 9.4. von Christoph Stamm, Politologe.

1 Offizielle Website: <www.itu.int/wsis>; Websites der beiden Gastländer: Schweiz, <www. wsisgeneva2003.org $>$; Tunesien, <www.smsitunis2005.org $>$.

$2<$ www.smsitunis2005.org>. Der Gedanke, unter der Ägide der Vereinten Nationen einen Gipfel mit der Beteiligung der Zivilgesellschaft und des Privatsektors zu organisieren, nahm auf verschiedenen Konferenzen allmählich Gestalt an, z.B. auf der Konferenz von Bamako im Jahr 2000, die von Akteuren aus Mali und aus Genf ausgerichtet wurde, <www.anais.org/SITES/BAM2000>. 
die nichtstaatlichen Akteure definierten, führten zu zähen Verhandlungen unter den Staaten und verursachten Spannungen zwischen den Regierungen und der Zivilgesellschaft.

Als Haupterfolg der ersten Phase ist zu werten, dass es gelungen ist, die Streitpunkte zwischen den Staaten und zwischen den verschiedenen vertretenen Akteuren zu erkennen. In Bezug auf die Grundsatzerklärung bereiteten hauptsächlich die Menschenrechte, die Meinungsäusserungsfreiheit und die Rolle der Medien Probleme. $\mathrm{Zu}$ diesen Fragen wurde mit grosser Mühe ein Konsens erzielt, was jedoch nicht für alle umstrittenen Fragen im Zusammenhang mit dem Aktionsplan der Fall war. Diese Streitthemen - besonders Gouvernanz des Internets und die Finanzierung - stehen in der zweiten Phase zur Diskussion. Ziel ist es, im Jahr 2005 in Tunis Beschlüsse zu fassen.

\section{Weltgipfel über die Informationsgesellschaft (WSIS)}

\section{Organisatoren}

Der Weltgipfel der Vereinten Nationen (WSIS) wurde unter der Schirmherrschaft von UNO-Generalsekretär Kofi Annan organisiert. Die Internationale Fernmeldeunion (ITU) war für die Durchführung der Vorbereitungen zuständig. Die Schweiz kümmerte sich als Gaststaat in der ersten Phase um die logistische und protokollarische Organisation und um die Sicherheit. Dafür investierte der Bund rund 20 Millionen Franken, davon 4 Millionen für die Sicherheit. Der Kanton Genf steuerte namentlich für das Exekutivsekretariat der Zivilgesellschaft und für die Sicherheit weitere 5 Millionen Franken bei.

Ort und Termin

Phase I: Genf, Palexpo, 10.-12. Dezember 2003.

Phase II: Tunis, 16.-18. November 2005.

\section{Ergebnisse der ersten Gipfelphase}

Die Staaten verabschiedeten zwei Dokumente:

- Politische Grundsatzerklärung (11 Seiten in 67 Punkten)a.

- Aktionsplan (19 Seiten in 29 Punkten)b.

Ferner wurden rund zehn sektorielle und/oder nicht-offizielle Unterlagen angenommen, so die Grundsatzerklärung der internationalen Zivilgesellschaft, die Erklärung der Städte und der lokalen Behörden auf dem Gipfel von Lyon (4.-5. Dezember)`', die Erklärung der indigenen Völker und die von den behinderten Personen verabschiedete Geneva Declaration on Accessible Information Society'.

\section{Beteiligung}

An der Genfer Phase beteiligten sich rund 11'000 Delegierte, welche 176 Staaten, 50 internationale Organisationen, 50 UNO-Unterorganisationen, 481 Nichtregierungsorganisationen (NG0), 98 Privatunternehmen und 631 Medienorgane vertraten. Daneben nahmen rund vierzig Staatschefs - mehrheitlich aus afrikanischen und arabischen Ländern - am Gipfel teile.

\section{Schweizerische Delegation}

Tripartite Delegation unter der Leitung von Bundesrat Moritz Leuenberger, Vorsteher des Eidgenössischen Departements für Umwelt, Verkehr, Energie und Kommunikation (UVEK), mit Vertretern des Bundesamts für Kommunikation (BAKOM), des Eidgenössischen Departements für auswärtige Angelegenheiten (EDA) und der Direktion für Entwicklung und Zusammenarbeit (DEZA), des Staatssekretariats für Wirtschaft (seco), des Bundesamts für Statistik (BFS), des Schweizerisches Bundesarchivs, des Bundesamts für Kultur (BAK), des Schweizerischen Instituts für Geistiges Eigentum, der Schweizerischen UNESCO-Kommission, von Impressum (ehemaliger Schweizer Verband der Journalistinnen und Journalisten), der Gewerkschaft Comedia, von Brot für alle und von economiesuissef.

a WSIS, Déclaration de principes, doc. WSIS-03/GENEVA/DOC/4-F, 12. Dezember 2003, <www.itu.int/wsis>.

WSIS, Aktionsplan, doc. WSIS-03/GENEVA/DOC/5-F, 12. Dezember 2003, <www.itu.int/wsis >.

Sommet mondial des villes et des pouvoirs locaux sur la société de l'information (Weltgipfel der Städte und der lokalen Behörden über die Informationsgesellschaft), <www.cities-lyon.org $>$. 
$<$ www.worldsummit2003.de>

e Zahlen der ITU. Aus den G-8-Mitgliedsländern war kein Staatschef zugegen. Auch der Privatsektor war nur schwach repräsentiert. Das Niveau der Beteiligung am WSIS ist deshalb besonders im Vergleich zum Gipfel von Rio, der 94 Staatschefs - darunter zahlreiche Vertreter der G-8 - versammelt hatte, als enttäuschend zu bewerten. Der schweizerische Delegationsleiter Marc Furrer gab zu, dass bei den Führern der nördlichen Länder eine gewisse Skepsis zu spüren sei, die hoffentlich überwunden werden könne („Die Schweiz hat ihr Ziel erreicht”, Swissinfo, 13. Dezember 2003). In Rio hatten sich über 7000 NGO versammelt. Ausserdem war in den Medien viel ausführlicher darüber berichtet worden.

f Die schweizerische Delegation war neben der deutschen und der kanadischen Delegation eine der wenigen, die auch Vertreter der Zivilgesellschaft am gesamten Vorbereitungsprozess beteiligte.

\section{$\square$ Die letzten Phasen des Vorbereitungsprozesses}

\section{PrepComs}

Der offizielle Gipfel mündete in einer Grundsatzerklärung und in einem Aktionsplan, der in langwierigen und konfliktreichen Diskussionen ausgearbeitet wurde. Am Ende des 3. Vorbereitungsausschusses (PrepCom, 15.-26. September 2003) standen die Verhandlungen weitgehend still, so dass eine zusätzliche Tagung organisiert werden musste (10.-14. November), die jedoch ebenfalls scheiterte. Die Schweizer Presse sprach vom Gipfel am Rand des Abgrunds ${ }^{3}$, vom Gipfel in der Sackgasse ${ }^{4}$ und vom vorprogrammierten Misserfolg 5 . Im Kreuzfeuer der Kritik stand nicht nur die Internationale Fernmeldeunion wegen ihrer politischen Schwäche, sondern auch die Schweiz als Gastland, der Dilettantentum ${ }^{6}$, mangelnde Führungsstärke und fehlende Vision ${ }^{7}$ vorgeworfen wurde.

\section{Kontroverse Punkte}

Die vier folgenden Fragen bildeten die grössten Stolpersteine:

- Menschenrechte. Bestimmte Länder - besonders China - lehnten in den Texten explizite Verweise auf die Universelle Menschenrechtserklärung, genauer auf Artikel 19 über die Meinungsäusserungsfreiheit, kategorisch ab. Zudem verlangten sie, dass die Staaten vom Imperativ der Menschenrechte abweichen und diese den nationalen Gesetzen unterstellen dürften.

- Finanzierung der Verringerung der digitalen Kluft. Der Aktionsplan verfolgt ehrgeizige und bezifferte Ziele zur Verringerung der Ungleichheiten im Zugang zu den Informations- und Kommunikationsmitteln: Bis zum Jahr 2015 sollen alle Dörfer, Universitäten, Primar- und Sekundarschulen, Gesundheitszentren, Krankenhäuser und öffentlichen Verwaltungen angeschlossen werden. Die Länder des Nordens - an der Spitze die Europäische Union - zogen die aktuelle Finanzierung (bilaterale Hilfe) und die öffentlich-privaten Partnerschaften vor; sie stemmten sich gegen den von den afrikanischen Ländern und den Ländern des Südens unterstützten Vorschlag des senegalesischen Präsidenten Abdoulaye Wade, einen digitalen Solidaritätsfonds einzurichten.

$\checkmark$ Medien. Weder die Anerkennung der Medien als wesentliche Akteure der Informationsgesellschaft, noch die Garantie der Freiheit, des Pluralismus und der Unabhängigkeit werden gewährleistet.

\footnotetext{
„Le Sommet de l'information au bord du gouffre“, Tribune de Genève, 11. November 2003. „Sommet mondial de l'information: l'impasse“, Le Temps, 15.-16. November 2003.

„A trois semaines de l'ouverture, le Sommet mondial de l'information court à l'échec“, Le Temps, 15.-16. November 2003.

6 „Une Suisse dilettante“, Le Temps, 12. September 2003.

7 „La Suisse au défi de son premier sommet onusien“, Le Temps, 12. September 2003.
} 
$\square$ Gouvernanz des Internets. Soll die Gouvernanz des Internets in der Hand der amerikanischen Privatfirma ICANN (Internet Corporation for Assigned Names and Numbers) bleiben, welche die Domain-Namen verwaltet? Soll diese Firma in mehrere private Unternehmen zergliedert werden? Soll ein geeignetes Organ innerhalb der UNO eingesetzt werden? Die Vereinigten Staaten, welche die ICANN de facto kontrollieren und in jeder (zwischen)staatlichen Intervention eine Gefahr für die Meinungsäusserungsfreiheit sehen, lehnten diesen Vorschlag ab; mehrere Regierungen, darunter China, sprachen sich dafür aus.

[D] Jahrbuch 2003, Nr. 2, Stéphane Koch, „Gouvernanz des Internets: eine beunruhigende Machtkonzentration“, S. 195-200, <www.iued-wsis.org>.

\section{Vermittlerrolle der Schweiz}

Die Schweiz, die zum ersten Mal als UNO-Mitglied einen Gipfel ausrichtete, unternahm neben der Rolle in der Logistik erhebliche diplomatische Anstrengungen und trat als „Vermittlerin“ auf, um einen Konsens zu verschiedenen heiklen Fragen herbeizuführen. Der ehemalige Bundesrat Adolf Ogi wurde Anfang November zum neuen Sondergesandten der Regierung beim WSIS ernannt.

Die Plattform comunica-ch ${ }^{8}$, die über vierzig Organisationen der schweizerischen Zivilgesellschaft umfasst, befürchtete, die schweizerische Delegation könnte - da sie wegen des Gipfels unter „Erfolgsdruck“ steht - in wesentlichen Punkten nachgeben. Aus diesem Grund legte sie dem Bundesrat, d.h. Präsident Pascal Couchepin und Aussenministerin Micheline Calmy-Rey, zwei Memoranden vor. In der Woche vor dem Gipfel hatte die Schweiz eine letzte Vorbereitungstagung, diesmal hinter verschlossenen Türen und ohne die Zivilgesellschaft, anberaumt.

\section{$\square$ Von den Staaten verabschiedete Dokumente}

Der eigentliche Erfolg des Gipfels von Genf besteht zweifellos darin, dass es gelungen ist, die Divergenzen unter den Staaten und unter den verschiedenen Akteuren zu einem derart breiten Thema überhaupt aufzuzeigen. Die Staaten erklärten sich einverstanden, unter der Ägide des Generalsekretärs der Vereinten Nationen Arbeitsgruppen zu verschiedenen umstrittenen Themen einzusetzen, damit im Jahr 2005 in Tunis Beschlüsse gefasst werden können. Die zweite Besonderheit des Gipfels betrifft die Verfahren: Die Zivilgesellschaft und der Privatsektor wurden formell als Partner in die internationale Diskussion eingebunden.

[D] Jahrbuch 2003, Nr. 2, Sean O’Siochrù und Bruce Girard, „Im System verstrickte Zivilgesellschaft“, S. 215-227, <www.iued-wsis.org>.

\section{Grundsatzerklärung}

Eine Einigung zur Grundsatzerklärung konnte schliesslich durch einen Minimalkonsens insbesondere zu folgenden strittigen Punkten erreicht werden:

- Menschenrechte. Das Risiko eines historischen Rückschlags in der Menschenrechtsfrage hat sich nicht bewahrheitet. Letztlich machte China Zugeständnisse. Die einführenden Artikel der Grundsatzerklärung enthalten einen ausdrücklichen Verweis auf die Universalität, Unteilbarkeit und Korrelation

$8<$ www.comunica-ch.net $>$. 
aller Menschenrechte und Grundfreiheiten. Die Erklärung nimmt Bezug auf die Universelle Menschenrechtserklärung, besonders auf Artikel 19 (Meinungsäusserungsfreiheit).

- Medien. Ein Absatz ist den traditionellen Medien (Presse, Radio und Fernsehen) gewidmet. Die Staaten bekräftigen darin ihr Engagement für die Grundsätze der Presse- und Informationsfreiheit, der Unabhängigkeit, des Pluralismus und der Vielfalt der Medien. Für dieses Prinzip hat sich die schweizerische Regierung unter dem Druck der Zivilgesellschaft sehr engagiert. China setzte im Gegenzug den Verweis auf eine verantwortungsvolle Informationsnutzung und -bearbeitung durch die Medien durch - gemäss anspruchsvollsten ethischen und professionellen Vorschriften, wie der Text zwar präzisiert, doch lässt dies verschiedene auch sehr restriktive Lesarten zu.

\section{Aktionsplan}

Der WSIS bildete den Schauplatz der traditionellen Auseinandersetzungen zwischen dem Norden und dem Süden zu den Finanzierungsfragen. Obwohl die Staaten gemeinsam private Investitionen zur Verringerung der digitalen Kluft generell in Betracht ziehen, erhoben sich abweichende Stimmen, welche die Glaubwürdigkeit des Marktes als wichtigstes Instrument zur Einebnung der technologischen Ungleichheiten in der Welt in Frage stellten.

Der senegalesische Präsident Abdoulaye Wade war Initiator und Hauptbefürworter des Projekts für einen digitalen Solidaritätsfonds. Dabei geht es darum, dass die internationale Völkergemeinschaft sich verpflichtet, alle Länder innerhalb einer „digitalen Schlange“ (durch eine obere und untere KonnektivitätsBandbreite definiert) zur Konvergenz heranzuführen'. Gemäss den Initianten liessen sich diese Anstrengungen mit einer Gebühr auf dem Verkauf von Informatikmaterial (z.B. 1 Dollar pro gekauftem oder verkauftem Computer) durch die Verbraucher des Nordens finanzieren.

[D] Jahrbuch 2003, Nr. 2, Marie Thorndahl, „Finanzierung und Wohlstandsteilung als Tabuthemen“, S. 207-213, <www.iued-wsis.org>.

Der Fonds wird als ,win-win“-Lösung (bei der alle nur gewinnen) für den Norden und für den Süden präsentiert; er sollte den Unternehmen des Nordens neue Märkte erschliessen und gleichzeitig die Bedürfnisse der zahlungsschwachen Bevölkerungen des Südens decken. Das Projekt besass mehrere Vorzüge, die eigentlich für eine Annahme durch die Staaten sprechen sollten: Der Fonds ist nicht obligatorisch (keine Gebühr, sondern ein freiwilliger Beitrag); er gehört vor den Hintergrund einer kapitalistischen Logik, die durch das Ankurbeln der Nachfrage im Süden das globale Wachstum fördern soll; er findet bei den meisten Ländern des Südens Rückhalt (Afrika, aber auch grosse Länder wie Indien oder Brasilien). Nichtsdestotrotz weigerten sich die Länder des Nordens, sich mit dem Fonds zu befassen, und argumentierten, dass die bereits bestehenden Finanzierungsmechanismen besser genutzt werden sollten. Der Meinungsunterschied wird in der Grundsatzerklärung anerkannt: Die Staaten nehmen den von einigen Staaten bekundeten Willen zur Kenntnis, einen „Internationalen digitalen Solidaritätsfonds" zu schaffen, der aus freiwilligen Beiträgen gespeist wird; sie nehmen auch den von anderen Staaten bekundeten

9 Abdoulaye Wade, Präsident des Senegals, Wortmeldung vom 17. Februar 2003, PrepCom 2, Genf, $<$ www.itu.int/wsis $>$. 
Willen zur Kenntnis, Studien über die existierenden Mechanismen sowie über die Effizienz und die Machbarkeit eines Fonds durchzuführen ${ }^{10}$.

Getreu einer der UNO eigenen Tradition des Kompromisses vertagten die Staaten den Beschluss auf den Gipfel von Tunis. Der Generalsekretär der Vereinten Nationen erhielt das Mandat, eine Arbeitsgruppe einzusetzen, die bis Dezember 2004 alle existierenden Fonds gründlich prüfen sollte. Die Ergebnisse dieser Arbeiten werden den Teilnehmern des Gipfels von Tunis vorgestellt ${ }^{11}$.

Die Städte und die lokalen Behörden - besonders Genf, Lyon und Turin kamen den Staaten zuvor und in die Quere, indem sie zum Abschluss des Gipfels ihre ersten Beiträge an den von Präsident Wade verfochtenen Fonds ankündigten. Ende Februar 2004 trug auch Dakar sein Scherflein bei, so dass der Fonds sich auf rund 2 Millionen Franken belief: 500'000 Franken von Genf, 300'000 Euro von Lyon und 400'000 Euro vom Senegal. Damit ist klar, dass der digitale Solidaritätsfonds de facto existiert. Genf hat sich als Sitzstadt anerboten. Bis zum Frühjahr 2004 sollte eine Stiftung nach Schweizer Recht geschaffen werden; Guy-Olivier Segond, der WSIS-Sonderbotschafter, wurde angefragt, diesen Fonds in der Anfangsphase zu leiten ${ }^{12}$. Neben der politischen Wirkung und dem Medienecho Die Städte ergreifen die Initiative! - bildet der Fonds eine gewisse Befriedigung für die Länder des Südens, die der internationalen Solidarität mit diesem Projekt Auftrieb verleihen wollten. Das eigentliche Novum ist jedoch nicht die Schaffung des Fonds, sondern dass die Städte und die lokalen Behörden engagiert auf der internationalen Bühne auftreten und damit in Sachen internationale Solidarität den Staaten das Zepter aus der Hand nehmen.

Unter den übrigen Themen, die bis zum Tunis-Gipfel aufgeschoben wurden, ist die Gouvernanz (Verwaltung und Regulierung) des Internets zu nennen. Gegenwärtig wird die Gouvernanz von privaten Stellen der reichen Länder, insbesondere von ICANN in den USA, gewährleistet. Einige Länder des Südens wünschen indessen eine stärkere Beteiligung der Vereinten Nationen oder anderer zwischenstaatlicher Stellen. Der Privatsektor und die Vereinigten Staaten, welche die ICANN de facto kontrollieren, lehnten es zuerst ab, sich mit dem Thema beschäftigen, während einige europäische Länder der ITU eine wichtigere Rolle erteilen möchten. Die Zivilgesellschaft schliesslich engagiert sich für die Schaffung kleiner, flexibler Strukturen, bei denen die drei „Gipfelpartner“ - die Staaten, der Privatsektor und die Zivilgesellschaft - ins Spiel kommen.

Kofi Annan wurde beauftragt, zu diesem Thema ebenfalls eine Arbeitsgruppe einzusetzen, welche die Gouvernanz des Internets sowie die jeweiligen Rollen und Verantwortungen definieren und Empfehlungen an die Adresse der Teilnehmer in Tunis 2005 ausarbeiten soll. Angesichts der Unklarheit, die das Thema umgibt, und in Anbetracht der polarisierten Positionen und widersprüchlichen Interessen bezweifeln viele Akteure, dass in Tunis ein echter Durchbruch in diesen Fragen erzielt werden kann.

Schliesslich ist zu erwähnen, dass zu einem entscheidenden Dossier, den geistigen Eigentumsrechten, eine Einigung zustande kam. Die Bezugnahme auf die

10 WSIS, Déclaration de principes, doc. WSIS-03/GENEVA/DOC/4-F, 12. Dezember 2003, S. 10, <www. itu.int/wsis>.

11 WSIS, Plan d'action, doc. WSIS-03/GENEVA/DOC/5-F, 12. Dezember 2003, S. 18, <www.itu. int/wsis >.

12 „Segond à la tête du Fonds de solidarité numérique ?“, Tribune de Genève, 26. Januar 2004. 
„,bestehenden“ Abkommen wurde gestrichen - gegen die Stellungnahme der Vereinigten Staaten und der Europäischen Union, welche diese Fragen nur an die Welthandelsorganisation (WTO) und die Weltorganisation für geistiges Eigentum (WIPO) delegieren wollten.

\section{$\square$ Offizielle Bilanz: Positive Bewertung und Genugtuung der Organisatoren}

Beim Abschluss der ersten Phase herrschte auf offizieller Seite generell Erleichterung und Zufriedenheit vor, obwohl die zahlreichen Hürden, die in den Verhandlungen und bei der Umsetzung des Aktionsplans noch zu überwinden sind, nicht verschwiegen wurden. Yoshio Utsumi, ITU-Generalsekretär und Generalsekretär des Gipfels, erwähnte, dass der Weltgipfel über die Informationsgesellschaft im Zeichen des Optimismus, im Geist des Konsenses und der Entschlossenheit ausgeklungen war, wies aber auch darauf hin, dass dieser Gipfel nur den Anfang eines langen und komplexen Prozesses bilde ${ }^{13}$. Der Berater von Kofi Annan, Nitin Desaï, bezeichnete das Ergebnis als das bestmögliche Resultat ${ }^{14}$. Der malische Präsident Amadou Toumani Touré begrüsste den Gipfel als Erfolg ${ }^{15}$. Der amerikanische Delegationsleiter David Gross pries die ausgezeichneten offiziellen Dokumente, welche die Ansichten der Vereinigten Staaten und der übrigen Welt widerspiegelten ${ }^{16}$.

Die schweizerische Bilanz des Gipfels ist aus zwei Dokumenten ersichtlich: zum einen aus dem Bericht der Koordinationsgruppe Informationsgesellschaft (KIG, <www.infosociety.ch>), zum anderen aus dem Bericht der schweizerischen Delegation am WSIS. Der Delegationsbericht umfasst eine detaillierte Bilanz der Resultate des Gipfels und seiner Bewertung durch die Schweiz. Er wird auf der Website des Bundesamtes für Kommunikation (BAKOM, <www. bacom.ch/de/amt/internationales/wsis $>$ ) verfügbar sein.

Marc Furrer, der Wortführer der Schweizer Delegation, zog eine relativ positive Bilanz. Die Schweiz habe ihre Sache gut gemacht ${ }^{17}$ und es sei ihr gelungen, ihre Prioritäten in Bezug auf die Inhalte durchzusetzen: Kampf gegen die Armut dank Informationstechnologien, Menschenrechte, Rolle der Medien, sprachliche Vielfalt. Moritz Leuenberger erwähnte in seiner Ansprache vor dem Plenum die Schwierigkeiten, die in den Verhandlungen aufgetreten waren, bekundete aber auch die allgemeine Zufriedenheit der Regierungen: „Noch vor zehn Tagen waren wir uns nicht einig [...] Heute schätze ich mich glücklich. Die Kompromisse, die wir geschlossen haben, sind brauchbar, auch wenn sie nicht allen unseren Erwartungen entsprechen [...] Der Weltgipfel über die Informationsgesellschaft in Genf gibt eine ausdrückliche politische Willensäusserung ab und seine Teilnehmer stellen sich hinter ein gemeinsames Anliegen: sie wollen die digitale Kluft überwinden, die kulturelle Vielfalt stärken, dem Recht auf freie Meinungsäusserung und der Pressefreiheit zum Durchbruch verhelfen. "18

13 ITU, Le SMSI encourage la solidarité et la création d'alliances mais il reste encore beaucoup à faire dans ce domaine, Pressemitteilung, Genf, 12. Dezember 2003, <www.itu.int/wsis>.

14 „Un plan d'action pour connecter la planète“, Le Monde, 15. Dezember 2003.

15 „Le Mali multiplie les pistes pour avoir accès à l'Internet“, Tribune de Genève, 16. Dezember 2003.

16 US-Aussenministerium, Mitteilung, 12. Dezember 2003.

17 „Die Schweiz hat ihr Ziel erreicht“, Swissinfo, 13. Dezember 2003.

18 Moritz Leuenberger, Ein weltumspannender politischer Wille, Rede an der Plenarsitzung des Weltgipfels über die Informationsgesellschaft, Genf, 11. Dezember 2003, <www.bakom.ch>. 
Neben dem diplomatischen Aspekt begrüssen die Regierungen auch den Erfolg der Parallelveranstaltungen. Eine Originalität des Genfer Gipfels bestand darin, dass er über 200 Ereignisse umfasste, die am Rande des offiziellen Programms von internationalen Organisationen, Staaten, Privatunternehmen und der Zivilgesellschaft ausgerichtet wurden ${ }^{19}$. Die Schweiz engagierte sich besonders in der Koordination und Finanzierung der Anlässe, welche den politischen Diskussionen einen konkreten und basisnahen Aspekt verleihen sollten.

Die sehr vielseitigen Ereignisse wurden mehr oder weniger als Kritik gegen den offiziellen Prozess organisiert. Am wichtigsten war die Ausstellung ICT for Development, welche von der DEZA in Zusammenarbeit mit Global Knowledge Partnership (GKP $)^{20}$ organisiert wurde. Das Event umfasste über 380 Projekte aus 80 Ländern. Unter den grossen Parallelforen sind ausserdem das World Electronic Media Forum (WEMF) ${ }^{21}$, an dem öffentliche und private Rundfunksender mitwirkten, sowie der Gipfel der Städte und lokalen Behörden über die Informationsgesellschaft in Lyon ${ }^{22}$ zu nennen. Im Zusammenhang mit den kritischen Beiträgen sind das World Forum on Communication Rights, das mit der Kampagne CRIS (Communication in the Information Society) ${ }^{23}$ eingeführt wurde, und das Community Media Forum zu erwähnen, das von den schweizerischen Hilfswerken Brot für alle und Fastenopfer zusammen organisiert wurde.

Die Presse äusserte sich nuancierter und gleichzeitig skeptischer. In den Kommentaren wurden die folgenden Punkte betont: zum einen die erfolgreiche Schadensbegrenzung und die Überwindung des Cancún-Syndroms, da eine erneute Konfrontation zwischen den Ländern des Südens und des Nordens zur Finanzierungsfrage vermieden werden konnte; zum anderen der unbestimmte und schwache Tenor der offiziellen Texte (als zaghafter Konsens ${ }^{24}$ und langer Katalog mit guten Absichten ${ }^{25}$ bezeichnet) sowie das besonders bei den Staaten des Nordens fehlende Engagement.

\section{$\square$ Kritische Bilanz der nichtstaatlichen Akteure}

\section{Verfahrensregeln und Beteiligung}

Die Zivilgesellschaft zieht eine gemischtere Bilanz. In Bezug auf die Beteiligung sind zwar nach hartem Ringen und langwierigen Diskussionen zu den Verfahrensregeln einige Verbesserungen und Neuerungen eingeführt worden. Die Nichtregierungskreise hatten formell noch nie in einem so hohen Mass an einem UNO-Gipfel mitgearbeitet: Während sie bislang in den Korridoren warten mussten oder auf Gegen-Gipfeln auf Distanz gehalten wurden, öffnete sich nun die Tür zum Verhandlungssaal einen Spalt breit. Bundespräsident Pascal Couchepin sagte dazu in seiner Eröffnungsansprache, dass die Zivilgesellschaft zum ersten Mal in der Geschichte der Vereinten Nationen eingeladen worden sei, in der Diskussion mitzureden; damit sei ein innovativer politischer Prozess in Gang gesetzt worden.

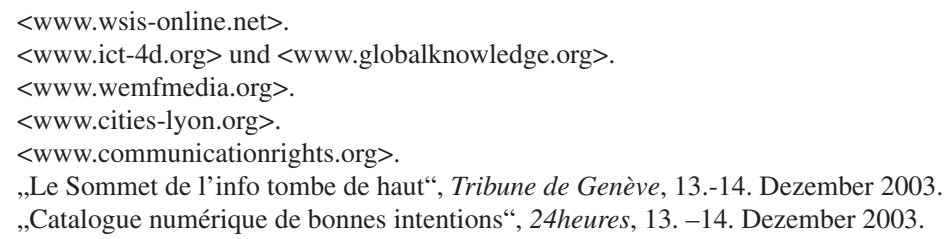


Konkret konnten sich die Vertreter der Zivilgesellschaft anlässlich der PrepComs jeweils im Plenum und zu Sitzungsbeginn der Unterarbeitsgruppen der Regierungen kurz zu Wort melden. Ausserdem konnten sie sich während des eigentlichen Gipfels am Podium äussern. Schliesslich wurde ein „Internationales Büro für die Zivilgesellschaft" ${ }^{\text {"26 }}$ geschaffen, das - strukturell auf der gleichen Ebene - mit den Regierungen die Verfahrensregeln erörtern soll. In der Schweiz wurde comunica-ch eng in den nationalen Konsultationsprozess und in die Arbeiten der Bundesversammlung einbezogen. comunica-ch war in der offiziellen Schweizer Delegation am WSIS mit drei Personen vertreten.

Dessen ungeachtet liegt der von den Organisatoren verheissene „,neue Gipfel“ noch in weiter Ferne. Die Organisatoren riefen zu einer aktiven und effektiven Beteiligung der Zivilgesellschaft auf und versprachen, neue Formen der Gouvernanz zu finden und die internationale Partnerschaft in einem echten tripartiten Prozess, an dem alle Spieler (Regierungen, zwischenstaatliche Organisationen, Unternehmen und Nichtregierungsorganisationen) mit gleich langen Spiessen mitmachen sollten, zu erneuern. Am rein beratenden Status der Zivilgesellschaft hat sich indessen nichts geändert. Die Beteiligung der Delegierten des Südens, besonders der ärmsten Länder, war stark eingeschränkt, da eine angemessene und ausreichende Finanzierung fehlte.

Der Gipfel blieb von Spannungen und offenen Konflikten zwischen den Behörden und den Organisationen der Zivilgesellschaft nicht verschont. In der Stadt Genf wurden mehrere Initiativen durchgeführt, die sich klar als Gegenentwürfe zum Gipfel positionierten, so insbesondere Polimedia Lab, die Konferenzen WSIS ? We Seize! $!^{27}$ und verschiedene Anti-WSIS-Veranstaltungen ${ }^{28}$.

\section{Inhalte: Alternative Erklärung}

Die Zivilgesellschaft konnte sich zwar zu Wort melden, hat aber den Eindruck, nicht wirklich Gehör zu finden. Obwohl die Erklärung weniger techniklastig ist, denkt die Zivilgesellschaft generell, dass gerade ihre innovativen Vorschläge und Forderungen mehrheitlich ignoriert wurden - mit dem Ergebnis, dass die Unterlagen Visionen und Massnahmen propagieren, welche die Interessen des Privatsektors denjenigen der Bevölkerungen überordnen und die Information in erster Linie als Ware und nicht als Recht oder als Gemeingut betrachten. Die

26 Der Beschluss, ein Internationales Büro für die Zivilgesellschaft einzurichten, wurde anlässlich der 2. PrepCom (17.- 28. Februar 2003) gefasst. Derzeit besteht das Büro aus 21 „Grossfamilien“: Gewerkschaften, Bildungs- und Universitätskreise, Vertreter der Wissenschaft und Technologie, Medien, Kulturschaffende und Kulturtätige, Städte und lokale Behörden, NGO, Jugendliche, Frauen, autochthone Völker, Behinderte, usw. Zum Büro gehören auch regionale Kontaktpunkte, <www.geneva2003.org>.

$27<$ www.geneva03.org>

28 Im Rahmen dieser Initiativen kam es besonders bei Demonstrationen und Kundgebungen in den Städten zu Zusammenstössen mit der Polizei. Beim Eingang zum Messegebäude Palexpo, in welchem der WSIS tagte, wurde kritisches Informationsmaterial (Trakte) beschlagnahmt. Die vom Gipfel ausgeschlossene NGO Reporters sans frontières richtete den Piratsender „Radio Non Grata“ ein, um den Gipfelgegnern eine Stimme zu verleihen (<www.radionongrata.info $>$ ). In der Palexpo fiel der ,Aktivismus" der tunesischen Delegierten auf, die Petitionen von Amnesty International und Exemplare der unabhängigen Zeitung Terra Viva verschwinden liessen und die von der Zivilgesellschaft organisierten kritischen Konferenzen unterwanderten (Terra Viva Online, <www.ipsnews.net/focus/tv society/indexfr.asp>; Rik Panganiban, Ralf Bendrath, How Was the Summit?, Genf/Berlin, 16. Dezember 2003, <www.worldsummit2003.de>). Presseartikel : „La police tord le cou à une manif qui n'en était pas une“, Le Courrier, 13. Dezember 2003; „Noté par ses participants, le SMSI récolte 7 sur 10“, Le Temps, 13. Dezember 2003. 
Staaten haben sich in keiner Weise konkret verpflichtet, die digitale Kluft zu überwinden. Darüber hinaus weisen die offiziellen Texte die folgenden gravierenden Mängel und Lücken auf:

$\checkmark$ Die für die Basisgemeinschaften der Entwicklungsländer wesentlich wichtigen Gemeinschaftsmedien werden mit keinem Wort erwähnt. Die schweizerische Delegation engagierte sich nachdrücklich für dieses Thema, scheiterte aber am Widerstand bestimmter, vor allem lateinamerikanischer, Länder.

- Die Förderung der freien Software mit offenem Quellcode - ein Schlüsselelement für den Aufbau einer einschliessenden, allen zugänglichen, den Bedürfnissen und kulturellen Besonderheiten der Entwicklungsländer entsprechenden Informationsgesellschaft - wurde nach zähen Diskussionen und etlichen Umformulierungen in letzter Minute im Konditionalis wieder eingeführt, ist aber praktisch inexistent. Offensichtlich hat der Wirtschaftssektor, angeführt von Microsoft und von der Internationalen Handelskammer (IHK), sein ganzes Gewicht in die Waagschale geworfen.

[D] Jahrbuch 2003, Nr. 2, Bernard Lang, „Der Kampf um die freie Software“, S. 201-205, <www.iuedwsis.org>.

Aus diesen Gründen beschloss die Zivilgesellschaft am Ende von PrepCom 3bis, die Verhandlungen aus Protest gegen den mangelnden politischen Willen der Staaten zu verlassen und statt dessen eigene Referenztexte auszuarbeiten und Parallelanlässe zum offiziellen Gipfel zu organisieren. So veröffentlichte sie einen Rahmen mit wesentlichen nicht verhandelbaren Punkten (Essential Benchmarks) und eine Alternativerklärung (Shaping Information Societies for Human Needs ${ }^{29}$. Diese Dokumente vermitteln die Vision einer ,alternativen“ sprich solidarischeren, freieren, demokratischeren und gerechteren - Informationsgesellschaft. Der Inhalt deckt sich im Übrigen weitgehend mit demjenigen der Grundsatzerklärung und des Aktionsplans von comunica-ch, welche ebenfalls das Ergebnis monatelanger Dialoge und Konsultationen innerhalb der schweizerischen Zivilgesellschaft sind.

In seiner Wortmeldung vor dem Plenum unterstrich Sean O'Siochrù, der Moderator der Kampagne CRIS, dass die Erklärung der Regierungen und diejenige der Zivilgesellschaft sich mit ihren Gegensätzen und Gemeinsamkeiten ergänzten; beide Texte enthielten einen Schlüsselsatz, welcher die Kommunikation als grundlegenden sozialen Prozess, als wesentliches menschliches Bedürfnis und als Basis der gesamten gesellschaftlichen Organisation beschreibe. Die Erklärung der Zivilgesellschaft gehe jedoch weiter und enthalte Vorschläge zum Aufbau einer Informations- und Kommunikationsgesellschaft, die den Menschen in den Mittelpunkt stelle.

\section{$\square$ Von Genf nach Tunis}

\section{Polemik um Tunesien}

Die Wahl von Tunis als Ausrichtungsort für die zweite Gipfelphase war stark umstritten. Andrés Allemand ${ }^{30}$ fragt sich, ob der zweite Gipfel angesichts der besonderen Rolle des Gaststaates ein Informations- oder ein Propagandagipfel

29 Dokumente einsehbar unter: <www.worldsummit2003.de/en/web/586.htm>.

30 „Le Sommet de l'info tombe de haut“, Tribune de Genève, 13.-14. Dezember 2003. 
würde. Tunesien, in Afrika ein Spitzenreiter in Sachen Kommunikationstechnologien und Entwicklung des Internets, wird allerdings von den NGO regelmässig wegen Verletzungen der Menschenrechte und der Meinungsäusserungsfreiheit sowie wegen der ausgeklügelten Kontrolle und Zensur über das Internet verurteilt. Der schweizerische Delegationsleiter Marc Furrer gab zu, dass die Tunesier mit einem Problem konfrontiert seien: Sie müssten nun reagieren, die Menschenrechte und die Pressefreiheit beachten und den Dialog mit der Zivilgesellschaft aufnehmen ${ }^{31}$.

In den letzten Monaten verschärfte sich die Polemik erneut, nachdem die Regierung Ben Ali die Ernennung von General Habib Hamar, dem die Weltorganisation gegen Folter (OMCT) Ausschreitungen vorwirft, zum Präsidenten des Vorbereitungsausschusses für die zweite Gipfelphase bekannt gab. Die Stadt Genf beispielsweise erklärte öffentlich, sie würde Tunis boykottieren, falls dieser Vorsitzende im Amt bliebe ${ }^{32}$.

Als Reaktion auf diese Vorwürfe geisselte der tunesische Aussenminister Habib Ben Yahia die Anschwärzungskampagne gegen das Image von Tunesien ${ }^{33}$. Shashi Tharoor, Vizegeneralsekretär der UNO, bezeichnete die Kritik an der Wahl Tunesiens als restriktive Sichtweise; es sei folgerichtig und symbolisch, dass nach der Schweiz, einem Industrieland des Nordens, Tunesien, ein Entwicklungsland des Südens, gewählt würde; man solle ein Land nicht nach seiner Vergangenheit beurteilen, sondern die Chance für Veränderungen am Schopf packen $^{34}$.

Verschiedene Menschenrechtsorganisationen verteilten Petitionen ${ }^{35}$, welche die Organisation des Gipfels von Tunis an vier Bedingungen knüpften: konkrete Beweise für den Willen der Regierung von Ben Ali, die Menschenrechtssituation zu verbessern; Rücktritt von General Hamar; Zusage von Tunis an alle Vertreter der Zivilgesellschaft, sich ungehindert an den Arbeiten des Gipfels beteiligen zu können; direkte Mitwirkung des UN-Hochkommissariats für Menschenrechte im Vorbereitungsprozess für Tunis.

Der Prozess geht weiter... aber vieles bleibt unklar

Ende Februar 2004 hatte die Schweiz noch keine offizielle Strategie für ihre Rolle in der zweiten Gipfelphase definiert. Die Schweiz kündigte an, sie sei bereit, in den Diskussionen mitzuwirken und Ratschläge zu erteilen; ihr Beitrag bestünde im Wesentlichen darin, über die interaktive Plattform <www.wsisonline.org > und die Teilfinanzierung des Konsultationsprozesses (z.B. Initiative open wsis) die Kontinuität zu gewährleisten. Durch die Unterstützung der Diskussionen solle verhindert werden, dass Tunis die Errungenschaften von Genf wieder rückgängig mache ${ }^{36}$.

Auch in Bern wird über darüber diskutiert, wie die Beteiligung der Zivilgesellschaft erleichtert werden kann. In Betracht kommt insbesondere die Unter-

\footnotetext{
31 „Que va faire la Suisse d'ici au sommet de Tunis en 2005 ?“, Le Temps, 13. Dezember 2003.

„La Tunisie à Genève: ,Une mascarade““, Le Matin, 5. Dezember 2003.

„Un plan d'action pour connecter la planète“, Le Monde, 15. Dezember 2003.

„Les Nations unies sont le miroir du monde“, Tribune de Genève, 16. Dezember 2003.

5 So insbesondere die Pétition des organisations de la société civile sur la Tunisie et le SMSI, 19. September 2003, <www.iris.sgdg.org/actions/smsi/hr-wsis/petition-tunisie-fr.html>.

36 „Que va faire la Suisse d'ici au sommet de Tunis 2005 ?“, Le Temps, 13. Dezember 2003.
} 
stützung an das Internationale Büro, welches einige als „UNO der NGO“ bezeichnen - ein Gremium der neuen globalen tripartiten Verhandlungen, in dessen Mittelpunkt Genf stehen könnte ${ }^{37}$.

Tunesien möchte den Schwerpunkt auf die Annahme einer ,digitalen Solidaritätscharta" legen, die auf die IKT-Anwendungen für die Entwicklung ausgerichtet ist.

Die tunesische Delegation schlug für den Prozess bis Tunis einen Zeitplan vor, der unter anderem zwei vorbereitende Konferenzen sowie thematische und regionale Konferenzen vorsah. Beim Abschluss des Gipfels legten die Staaten zwar keinen Terminplan fest, kündigten aber an, dass im Internationalen Büro eine ständige Arbeitsgruppe für die Vorbereitungen für Tunis geschaffen werden solle.

Ende Februar 2004 existieren die beiden Arbeitsgruppen - über die Finanzierung und über die Gouvernanz des Internets - immer noch nicht. Die Vorstellungen zu Tunis 2005 bleiben sehr unklar. Wird Tunis zu einer Konferenz, welche die in Genf ungelösten Fragen klären soll? Eine Konferenz, die neue Texte ausarbeitet, oder wie andere UNO-Gipfel eine Konferenz über die Umsetzung des Aktionsplans? Das Fehlen klarer Zielvorgaben, gepaart mit dem Zeitdruck, wirft einen langen Schatten auf das Gelingen der zweiten Phase.

\section{QUELLEN}

\section{Dokumente}

WSIS, Déclaration de principes, doc. WSIS-03/GENEVA/DOC/4-F, 12. Dezember 2003, <www.itu.int/ wsis $>$.

WSIS, Plan d'action, doc. WSIS-03/GENEVA/DOC/5-F, 12. Dezember 2003, <www.itu.int/wsis>.

Erklärung der Zivilgesellschaft anlässlich des WSIS, Définir des sociétés de l'information centrées sur les besoins des êtres humains, Genf, 11. Dezember 2003, <www.worldsummit2003.de>.

\section{Reden}

Abdoulaye Wade, Präsident des Senegals, Ansprache vom 17. Februar 2003, PrepCom 2, Genf, $<$ www.itu.int/ wsis $>$.

Leuenberger Moritz, Ein weltumspannender politischer Wille, Rede an der Plenarversammlung des WSIS, Genf, 11. Dezember 2003, <www.bakom.ch>.

\section{Artikel und Pressemitteilungen}

Le Courrier, „La police tord le cou à une manif qui n’en était pas une“, 13. Dezember 2003.

Le Matin, „La Tunisie à Genève: ,Une mascarade““, 5. Dezember 2003, S. 2.

Le Monde, „Un plan d'action pour connecter la planète“, 15. Dezember 2003, S. 5.

Le Temps, „Genève sera à l'information ce que Rio a été pour l'environnement“, „La Suisse au défi de son premier sommet onusien“, „Une Suisse dilettante“, 12. September 2003; „A trois semaines de l'ouverture, le Sommet mondial de l'information court à l'échec“, „Sommet mondial de l'information: l'impasse“, 15.-16. November 2003; „Noté par ses participants, le SMSI récolte 7 sur 10“, „Que va faire la Suisse d'ici au sommet de Tunis en 2005 ?“, 13. Dezember 2003.

Panganiban Rik, Bendrath Ralf, How Was the Summit?, Genf/Berlin, 16. Dezember 2003, <www. worldsummit2003.de>.

Swissinfo, „Die Schweiz hat ihr Ziel erreicht“, 13. Dezember 2003.

Tribune de Genève, „Le Sommet de l'information au bord du gouffre“, 11.November 2003; „Le Sommet de l'info tombe de haut“, 13.-14. Dezember 2003; „Le Mali multiplie les pistes pour avoir accès à l'Internet“, „Les Nations unies sont le miroir du monde“, 16. Dezember 2003; „Segond à la tête du Fonds de solidarité numérique ?“, 26. Januar 2004; „Genève saura-t-elle ancrer ,l'ONU des ONG“?", 7.-8. Februar 2004.

ITU, Le SMSI encourage la solidarité et la création d'alliances mais il reste encore beaucoup à faire dans ce domaine, Pressemitteilung, Genf, 12. Dezember 2003.

24heures, „Catalogue numérique de bonnes intentions“, 13.-14. Dezember 2003.

37 „Genève saura-t-elle ancrer ,l'ONU des ONG“ ?“ Tribune de Genève, 7.-8. Februar 2004 


\section{INTERNET-ADRESSEN}

Bundesamt für Kommunikation (BAKOM) : <www.bakom.ch $>$. infosociety.ch, eine Initiative des Bundes: <www.infosociety.ch>.

Internationale Fernmeldeunion (ITU), offizielle Website des WSIS : <www.itu.int/wsis>.

Website der Schweiz (Gastland der ersten Phase) : <www.wsisgeneva2003.org $>$.

Website Tunesiens (Gastland der zweiten Phase) : <www.smsitunis2005.org $>$.

Exekutivsekretariat, Abteilung Zivilgesellschaft: <www.geneva2003.org >.

Global Knowledge Partnership: <www.globalknowledge.org $>$.

ICT4D Plattform: <www.ict-4d.org>.

Pétition des organisations de la société civile sur la Tunisie et le SMSI, 19. September 2003 : <www.iris. sgdg.org/actions/smsi/hr-wsis/petition-tunisie-fr.html>.

Plattform der Zivilgesellschaft der Heinrich-Böll-Stiftung : <www.worldsummit2003.de>.

Schweizerische Plattform für die Informationsgesellschaft : <www.comunica-ch.net $>$.

Reporters sans frontières, Radio Non Grata : <www.radionongrata.info $>$.

Weltgipfel der Städte und der lokalen Behörden über die Informationsgesellschaft: $<w w w . c i t i e s-l y o n . o r g>$.

World Electronic Media Forum : <www.wemfmedia.org $>$.

World Forum on Communication Rights : <www.communicationrights.org $>$.

WSIS Community Platform: <www.wsis-online.net>.

WSIS ? We Seize ! : <www.geneva03.org>.

\subsection{UNESCO - Organisation der Vereinten Nationen für Erziehung, Wissenschaft und Kultur}

Die 32. Generalkonferenz der UNESCO fand vom 29. September bis zum 17. Oktober 2003 in Paris statt. Die Konferenz stand im Zeichen der Rückkehr der Vereinigten Staaten, die nach rund zwanzig Jahren der Organisation wieder beitraten. Unter den wichtigen Konferenzbeschlüssen ist das Projekt für die Ausarbeitung eines Übereinkommens über die kulturelle Vielfalt zu nennen, das trotz der Ablehnung bestimmter Staaten, darunter die Vereinigten Staaten, per Konsens verabschiedet wurde. Die Schweiz wurde in den Exekutivrat gewählt - ein beachtlicher Erfolg für die Politik des Bundesrates zur Stärkung der schweizerischen Präsenz in den Institutionen der Vereinten Nationen.

\section{$\square$ Anfänge}

Die Abkürzung UNESCO ${ }^{38}$ steht für „Organisation der Vereinten Nationen für Erziehung, Wissenschaft und Kultur“. Die UNESCO wurde nach dem Zweiten Weltkrieg ins Leben gerufen, als die kriegsverwüsteten Länder eine Reihe von Problemen dringend bewältigen mussten: Wiederaufbau der durch die feindliche Besatzung zerstörten Ausbildungssysteme, Wiedereröffnung der Museen und Bibliotheken, deren Bestände geplündert und verstreut worden waren, usw. Zwischen 1942 und 1945 tagte die Konferenz der Erziehungsminister der Alliierten mehrmals in London, um die Grundlagen zur Errichtung einer internationalen Erziehungsorganisation zu schaffen. Die Konferenz von London, an der die Vertreter von 44 Regierungen und internationalen Institutionen mitwirkten, erstellte und verabschiedete am 16. November 1945 die UNESCO-Gründungsakte. Die eigentliche Geburtsstunde der Organisation schlug am 4. November 1946, nachdem zwanzig Signatarstaaten ${ }^{39}$ ihre Ratifizierungsurkunde hinterlegt hatten. In

38 United Nations Educational, Scientific and Cultural Organization.

39 Ägypten, Australien, Brasilien, China, Dänemark, Dominikanische Republik, Frankreich, Griechenland, Indien, Kanada, Libanon, Mexiko, Neuseeland, Norwegen, Saudi-Arabien, Südafrika, Tschechoslowakei, Türkei, Vereinigte Staaten von Amerika, Vereinigtes Königreich. 
den darauf folgenden Jahren traten die Staaten nach und nach der UNESCO bei. Bemerkenswert sind zwei „Beitrittswellen“ im Zusammenhang mit epochalen Ereignissen : 1960 stiessen mehrere ehemalige Kolonien als unabhängige Staaten zur UNESCO; 1992 wurde die UdSSR (seit 1954 Mitglied) von der Russischen Föderation abgelöst und von den zwölf Nachfolgestaaten der Sowjetunion gefolgt, die der UNESCO zwischen 1991 und 1993 beitraten ${ }^{40}$.

\section{$\square$ Krise und Reformen}

$\mathrm{Ab}$ den 70er Jahren war die Organisation anhaltenden Spannungen ausgesetzt. Die Zeit war von der lebhaften Diskussion um die israelische Politik in den besetzten Gebieten geprägt. Diese Auseinandersetzung veranlasste die Vereinigten Staaten, die Beitragszahlungen zwei Jahre lang auszusetzen. Nach dem Beschluss der Vereinten Nationen über die "Neue Weltwirtschaftsordnung“ (NWWO) befasste sich die UNESCO mit einem Projekt über die „Neue Weltinformations- und Kommunikationsordnung" (NWIKO), welches in der westlichen Presse Besorgnis weckte und auch die Regierungen alarmierte. In allen Bereichen wurden die Forderungen der Dritten Welt von den kommunistischen Ländern hochgeschaukelt. Um zu vermeiden, dass die westlichen Länder in den Abstimmungen über die Entschliessungen in eine Minderheitsposition gedrängt wurden, wurde die Praxis des Konsenses eingeführt. Die voranschreitende Politisierung der UNESCO stiess jedoch bei den Industrieländern auf zunehmendes Missfallen. Ausserdem monierten die angelsächsischen Länder die Missstände in der Verwaltung der Organisation, die aufgeblähte Bürokratie und die mangelnde Haushaltsdisziplin. Die Krise gipfelte im Austritt der Vereinigten Staaten 1984 sowie im Rückzug des Vereinigten Königreichs und Singapurs $1985^{41}$. Seit seiner Wahl im Jahr 1999 hat der amtierende Generaldirektor Koichiro Matsuura jedoch zahlreiche Reformen - insbesondere struktureller Art - eingeleitet, welche das Vereinigte Königreich und die Vereinigten Staaten zur Rückkehr in die Organisation bewogen haben.

\section{$\square$ Auftrag der UNESCO}

Das Hauptziel der UNESCO besteht darin, durch Förderung der Zusammenarbeit zwischen den Völkern auf den Gebieten der Erziehung, Wissenschaft, Kultur und Kommunikation zur Wahrung von Frieden und Sicherheit in der Welt beizutragen, um die allgemeine Achtung des Rechts und der Gerechtigkeit, der Menschenrechte und Grundfreiheiten für alle ohne Unterscheidung nach Rasse, Geschlecht, Sprache oder Religion zu stärken, welche die Charta der Vereinten Nationen allen Völkern zuerkennt.

Die UNESCO unterstützt die Mitgliedsstaaten mit verschiedenen, in ihren Kompetenzbereich fallenden Projekten: Sie organisiert Bildungskurse, besorgt die notwendigen Ausrüstungen, gewährt Stipendien, stellt Experten und Ermittlungsbeauftragte zur Verfügung, leistet finanzielle Hilfe, unterstützt die Schaffung von Forschungsnetzen, führt Untersuchungen durch, usw. Dennoch ist die UNESCO keine eigentliche Entwicklungsorganisation. Sie sieht sich z.B. nicht dazu berufen, Grossprojekte zur Verbesserung der Infrastruktur anzugehen. Ihre

40 Crucifix Isabelle (Dir.), Organisations internationales à vocation universelle, Paris, La Documentation Française, 1993.

41 Ibid. 
Unterstützung ist vor allem intellektueller Natur: Sie stellt Beratungsdienste und Fachleute zur Verfügung ${ }^{42}$. Unter den wichtigen Aktivitäten der Organisation im Jahr 2003 ist zu erwähnen, dass die UNESCO mit der Koordination sämtlicher Tätigkeiten der UNO im Zusammenhang mit dem Internationalen Jahr des Süsswassers beauftragt wurde ${ }^{43}$.

Jahrbuch 2004, Nr. 1, Kap. 10.4. „Internationales Jahr des Süswassers 2003“.

\section{$\square$ Struktur der UNESCO}

Die UNESCO besitzt eine komplexe Struktur mit zwei Lenkungsorganen, einem Sekretariat und nationalen Kommissionen.

Die Lenkungsorgane sind:

- die Generalkonferenz, das Hauptentscheidungsgremium. Die Delegierten der 190 Mitgliedsstaaten treten alle zwei Jahre zusammen und beschliessen über das Programm und den Zweijahreshaushalt der Organisation;

$\checkmark$ der Exekutivrat, der sich aus 58 von der Generalkonferenz gewählten Vertretern zusammensetzt. Er tagt zweimal jährlich und überwacht die Durchführung der Programme, die angemessene Umsetzung der entsprechenden Haushaltsposten, die Befolgung der Beschlüsse der Generalkonferenz und bereitet die Tagungen der Konferenz vor.

Das Sekretariat der Organisation mit Sitz in Paris ist für die Durchführung der Projekte und für Verwaltungsaufgaben zuständig. Es ist dem für sechs Jahre gewählten Generaldirektor (gegenwärtig der Japaner Koichiro Matsuura) unterstellt. Das Sekretariat umfasst fünf Sektoren, die mit der Umsetzung der Tätigkeiten in den Zuständigkeitsbereichen der UNESCO beauftragt sind: Erziehung; Naturwissenschaften; Geistes- und Sozialwissenschaften; Kultur; Kommunikation, Information und Informatik.

Die nationalen Kommissionen spielen als Konsultativgremien und als Verbindungsstellen zwischen der Organisation und dem Zielpublikum der UNESCOTätigkeiten in den Mitgliedsstaaten eine Schlüsselrolle.

\section{$\square$ Finanzierung der UNESCO}

Die UNESCO finanziert ihre Tätigkeiten hauptsächlich aus den Pflichtbeiträgen ihrer Mitgliedsstaaten. Der reguläre Zweijahreshaushalt 2002-2003 betrug rund 544 Millionen US-Dollar (rund 918 Millionen Schweizer Franken). Zusätzlich erhält die Organisation ausserordentliche Beiträge, Treuhandgelder für bestimmte Projekte sowie Mittel anderer UNO-Einrichtungen, namentlich vom Entwicklungsprogramm der Vereinten Nationen (UNDP) und vom Bevölkerungsfonds der Vereinten Nationen (UNFPA), von der Weltbank und den regionalen Entwicklungsbanken sowie freiwillige Beiträge von Mitgliedsstaaten und Spenden (insbesondere für Bildungshilfe in Konfliktregionen). Der ordentliche Haushalt der UNESCO liegt derzeit in realen Zahlen (d.h. inflationsbereinigt) deutlich unter dem Niveau der 70er Jahre.

42 Nationale Schweizerische UNESCO-Kommission. Die Schweiz und die UNESCO. Fragen und Antworten, Bern, 1991, S. 15.

43 Offizielle Website des Internationalen Süsswasserjahres : <www.wateryear2003.org>. 
Mit rund 6,5 Millionen Franken pro Jahr gehört die Schweiz zu den zwölf bedeutendsten Beitragszahlern der Organisation. Spitzenreiter sind Japan (Anteil am ordentlichen Haushalt: $25 \%$ ) und Deutschland (13,4\%), gefolgt von Frankreich (9\%), Italien (7,5\%), Kanada (4\%) und Spanien (3,5\%). Die Schweiz belegt mit 1,2 Prozent den zwölften Rang ${ }^{44}$.

\section{$\square$ Die Schweiz und die UNESCO}

Der UNESCO-Beitritt der Schweiz im Jahr 1948 erfolgte im Rahmen der Politik des Bundesrates für die internationalen Zusammenarbeit. In seiner Botschaft an die Bundesversammlung über den Beitritt der Schweiz zur UNESCO unterstrich der Bundesrat, „dass sich unser Land politisch und geistig isolieren würde, wenn es nicht an einer Organisation mitarbeitete, deren Ziele offensichtlich mit den Bestrebungen im Einklang stehen, die es selbst auf nationalem Boden und in seinen Beziehungen mit den anderen Ländern zu verwirklichen sucht. Nach der Isolierung der Kriegszeit liegt es in seinem Interesse, mit Nachdruck jede Gelegenheit zu ergreifen, um seinen Horizont zu erweitern, um mit anderen Kulturen in Kontakt zu treten und an den Bemühungen mitzuwirken, die zum Ziele haben, die Völker einander näherzubringen und sie zu lehren, eine andere Sprache zu sprechen als diejenige der Furcht, des Hasses oder der Gewalt" ${ }^{\star 45}$.

\section{Die Schweizerische UNESCO-Kommission}

Im Jahr 1949 setzte der Bundesrat die Nationale Schweizerische UNESCOKommission (NSUK) ein und erteilte ihr vier Aufgaben:

๑ sie ist das Konsultationsorgan der Bundesbehörden für die Beziehungen der Schweiz zur UNESCO;

- sie ist die Verbindungsstelle zwischen der UNESCO und den entsprechenden schweizerischen Kreisen, die sich mit Erziehung, Wissenschaft, Kultur und Kommunikation befassen;

口 sie sensibilisiert die interessierten Kreise und die Öffentlichkeit für die wesentlichen Fragen, mit denen sich die UNESCO beschäftigt;

๑ sie setzt in den Tätigkeiten der Sektionen die UNESCO-Programme um und beaufsichtigt ihre Durchführung.

Derzeit zählt die NSUK 30 Mitglieder, die für einen Zeitraum von vier Jahren vom Bundesrat ad personam ernannt werden. Die NSUK gehört anerkanntermassen zu den aktivsten nationalen Kommissionen ${ }^{46}$. Ihr Sekretariat ist dem Eidgenössischen Departement für auswärtige Angelegenheiten (EDA) angegliedert.

\section{$\square$ 32. UNESCO-Generalkonferenz}

Die 32. Generalkonferenz der UNESCO tagte vom 29. September bis zum 17. Oktober 2003 am Sitz der Organisation in Paris. Sie verabschiedete fünf normensetzende $\mathrm{Texte}^{47}$, darunter das Internationale Übereinkommen über die Wahrung des immateriellen Kulturerbes und die Internationale Erklärung über

44 Finanzierung der UNESCO : <www.unesco.ch/organ-d/finanzierung_frame.htm>

45 Schweizerische UNESCO-Kommission, op. cit., S. 22.

46 Website der Nationalen Schweizerischen UNESCO-Kommission : <www.unesco.ch>.

47 Für nähere Informationen über die verabschiedeten Texte wird auf die Website der UNESCO verwiesen: <www.unesco.org >. 
genetische Daten des Menschen. Die Konferenz verabschiedete ferner ein Programm mit folgenden Prioritäten: Grundausbildung für alle; Wasser und damit verbundene Ökosysteme; Ethik der Wissenschaft und Technologie; Förderung der kulturellen Vielfalt und des Dialogs unter den Kulturen; Zugang zu Information und Wissen. Die Rückkehr der Vereinigten Staaten in die UNESCO stellte einen Meilenstein in der Geschichte der Organisation dar und erlaubte es ihr, einen höheren Haushalt 2004-2005 (610 Millionen Dollar) zu beschliessen. Die Vereinigten Staaten wurden im Übrigen seit ihrem Wiedereintritt in den Exekutivrat gewählt.

\section{$\square$ Entwurf eines internationalen Übereinkommens über die kulturelle Vielfalt}

Nach einer lebhaften Diskussion verabschiedete die Generalkonferenz per Konsens den Entwurf für die Ausarbeitung einer internationalen normensetzenden Urkunde über die kulturelle Vielfalt. Im Jahr 2001 hatte sie die Allgemeine Erklärung zur kulturellen Vielfalt angenommen ${ }^{48}$, welche erstmals die kulturelle Vielfalt als „gemeinsames Erbe der Menschheit“ und ihre Verteidigung als einen ethischen Imperativ anerkannte, der untrennbar mit der Achtung der Menschenwürde verknüpft ist.

Die Delegierten kamen überein, dass der Schutz der Vielfalt der kulturellen Inhalte und der künstlerischen Ausdrucksformen Gegenstand eines internationalen Übereinkommens bilden sollte. Ausserdem erinnerten sie daran, dass Gespräche mit der Welthandelsorganisation (WTO), der Konferenz der Vereinten Nationen für Handel und Entwicklung (UNCTAD) und der Weltorganisation für geistiges Eigentum (WIPO) geführt werden müssen. Die Resolution appelliert an den Generaldirektor, der Generalkonferenz an ihrer nächsten Tagung im Herbst $2005^{49}$ einen Vorentwurf zu einem Übereinkommen über den Schutz der kulturellen Inhalte und der künstlerischen Ausdrucksformen zu unterbreiten.

Das Übereinkommen soll insbesondere das Recht der Staaten verkünden, eine Kulturpolitik durchzuführen und die Produktion im Kulturbereich zu fördern und zu schützen. Zu diesem Zweck sollen Konzept und Besonderheiten der Kulturgüter und des Kulturgüterhandels definiert werden, welche - auch in der WTO $^{50}$ - rechtlich massgebend sind.

Auf der Seite der Hauptinitianten des Entwurfs, Frankreich und Kanada, stehen rund fünfzig Mitgliedsstaaten der Internationalen Organisation der Frankophonie. Auch die Gruppe der 77 spricht sich für den Entwurf eines Übereinkommens aus. Die Europäische Union dagegen ist in dieser Frage gespalten: Spanien macht Vorbehalte geltend, während das Vereinigte Königreich, die Niederlande und bestimmte skandinavische Länder, wie im Übrigen auch Australien, Neuseeland und Indien, Ablehnung signalisieren. Die Schweiz setzt sich aktiv für die Idee eines einschlägigen Übereinkommens ein. Der Direktor des Bundesamts für Kultur, David Streiff, unterstreicht die Bedeutung des schweizerischen Engagements: Kanada und Frankreich seien die Lokomotiven, aber die Schweiz folge unmittelbar danach. Die Schweiz teile die Überzeugung, dass die

48 Auf der UNESCO-Website einsehbar (deutsch und französisch): <www.unesco.ch>, > UNESCO, > Dokumente.

49 UNESCO, Pressemitteilung, Paris, 17. Oktober 2003.

${ }^{50}$ „De retour à l'UNESCO, Washington lance l'offensive contre le projet de convention sur la diversité culturelle", Le Monde, 30. September 2003. 
Kultur kein beliebiges Gut sei, und verteidige die Wahrung und den Schutz der kulturellen Vielfalt. Auch das Staatssekretariat für Wirtschaft (seco) teilt diese Position, die von Bundesrätin Micheline Calmy-Rey anlässlich ihrer Ansprache am 1. Oktober $2003^{51}$ offiziell verkündet wurde.

\section{$\square$ Wahl der Schweiz in den Exekutivrat der UNESCO}

Die Schweiz ist seit 1948 relativ regelmässig im Exekutivrat vertreten ${ }^{52}$. Anlässlich der Generalkonferenz von 2003 wurde sie für eine Dauer von vier Jahren gewählt. Mit der Einsitznahme in den Exekutivrat verstärkt die Schweiz ihren Einsatz auf dem Gebiet der Ausgestaltung und Umsetzung der Ziele und Prioritäten der UNESCO. Im Vordergrund des schweizerischen Engagements stehen die „Bildung für alle/Education pour tous“, die Förderung der Wissensgesellschaft, die Stärkung der Rolle der UNESCO auf dem Gebiet des Dialogs der Zivilisationen, Kulturen und Religionen, der Schutz und die Erhaltung des Welterbes sowie die Weiterentwicklung des Völkerrechts, namentlich im Bereich der kulturellen Vielfalt ${ }^{53}$. Ausserdem wurde die Schweiz in verschiedene Unterorgane gewählt, darunter das Intergouvernementale Komitee für die Förderung der Rückkehr von Kulturgütern in ihr Herkunftsland oder ihrer Rückgabe im Falle illegaler Aneignung (ICPRBC), in dem sie erstmals vertreten ist. Darüber hinaus wurde sie in den Rat des Internationalen Büros für Erziehung (BIE) gewählt ${ }^{54}$.

\section{$\square$ Position der Schweiz}

Bundesrätin Micheline Calmy-Rey, Vorsteherin des Eidgenössischen Departements für auswärtige Angelegenheiten (EDA), nahm am 1. Oktober 2003 an der Generalkonferenz teil. In ihrer offiziellen Ansprache ${ }^{55}$ erinnerte sie an das Engagement der Schweiz zu verschiedenen Punkten. Mit Blick auf den Weltgipfel über die Informationsgesellschaft (WSIS) betonte die Bundesrätin, dass die Schweiz den Entwurf einer Charta zum Schutz des digitalen Erbes und den Entwurf einer Empfehlung zur Förderung und Nutzung der Mehrsprachigkeit und des universalen Zugangs zum Cyberspace vorbehaltlos unterstütze. Ausserdem erwähnte sie das Interesse der Schweiz an der Allgemeinen Erklärung über die kulturelle Vielfalt im Jahr 2001 und bestätigte die Unterstützung für die Ausarbeitung einer verbindlichen Urkunde für den Schutz und die Förderung der kulturellen Vielfalt (siehe oben ,Entwurf eines internationalen Übereinkommens über die kulturelle Vielfalt"). Im Erziehungsbereich bekräftigte sie das Engagement zugunsten des in Genf angesiedelten Internationalen Büros für Erziehung (BIE), dem finanzielle und personelle Ressourcen zugesichert werden. Hingegen forderte die Bundesrätin die UNESCO nachdrücklich auf, das BIE mit den zur Erfüllung seiner Aufgaben erforderlichen Finanzmitteln und der notwendi-

51 „David Streiff: ,La Suisse a beaucoup à apporter““, Le Temps, 15. Oktober 2003.

52 Seit der Gründung der UNESCO hatte die Schweiz siebenmal einen Sitz im Exekutivrat. Sie war durch folgende Personen vertreten: J. Piaget (1950-1954), B. Barbey (1964-1970), J. Hersch (19701972), Ch. Hummel (1976-1980), F. Muheim (1987-1991), D. Morf (1994-1997). D. Feldmeyer wird die Schweiz im Zeitraum 2003-2007 vertreten.

53 UNESCO Info. Newsletter der Schweizerischen UNESCO-Kommission, Nr. 17, Oktober 2003.

54 Eidgenössisches Departement für auswärtige Angelegenheiten, Die Schweiz nimmt Einsitz in drei Unterorgane der UNESCO, Pressemitteilung, 14. Oktober 2003.

55 Wortmeldung von Bundesrätin Micheline Calmy-Rey anlässlich der 32. UNESCO-Generalkonferenz, Paris, 1. Oktober (einsehbar auf der Website des EDA <www.eda.admin.ch>). 
gen Autonomie auszustatten. Im Bereich des Schutzes des Kulturerbes wurde das Engagement der Schweiz für die Wiederherstellung des afghanischen Kulturerbes sowie die internationalen Anstrengungen für die Erhaltung und Rückerstattung der Kulturgüter des Iraks erwähnt. Im Rahmen des Besuchs bei der UNESCO überreichte Micheline Calmy-Rey dem Generaldirektor die schweizerische Ratifizierungsurkunde zur Konvention von 1970 über den unzulässigen Kulturgütertransfer.

[D] Jahrbuch 2004, Nr. 1, Kap. 13, „Gesetzgebung über den Kunsthandel in der Schweiz“.

\section{$\square$ Rückkehr der Vereinigten Staaten in die UNESCO}

Die 32. Generalkonferenz stand im Zeichen der Rückkehr der Vereinigten Staaten in die UNESCO nach fast zwanzigjähriger Abwesenheit. 1984 hatten die Vereinigten Staaten unter der Präsidentschaft von Ronald Reagan die UNESCO verlassen, aus Protest gegen das, was sie als Misswirtschaft und wachsende Politisierung der Organisation mit eindeutig antiwestlichen Tendenzen ansahen. Die Entscheidung von Präsident Bush, der Organisation erneut beizutreten, rechtfertigt sich gemäss der amerikanischen Regierung durch die umfassenden Veränderungen, die seither stattgefunden haben. Nach Auffassung der Vereinigten Staaten setzt sich die UNESCO erneut für die Förderung von Grundwerten wie Pressefreiheit und Bildung für alle ein. Ausserdem weisen die Vereinigten Staaten auf die weit reichenden Bemühungen des Generaldirektors im Verwaltungsbereich hin (strikte Personalpolitik, Schaffung einer internen Kontrollstelle, Einführung einer Haushalts- und Managementsoftware, Dezentralisierung der bürokratischen Struktur $)^{56}$.

Dagegen stellen zahlreiche Beobachter fest, dass die Rückkehr der Vereinigten Staaten in die UNESCO mit dem Entwurf für die Ausarbeitung eines Übereinkommens über die kulturelle Vielfalt zusammenfällt: Dieses Übereinkommen soll die Anerkennung der „Ausnahmeregelung für die Kultur“, welche die USA und die Europäische Union, vor allem Frankreich, in den Verhandlungen der Welthandelsorganisation (WTO) spaltet, in verbindlichen Rechtsnormen verankern. Die Frage ist für die USA von grossem Gewicht, zumal die Filmindustrie sich zum wichtigsten Exportposten entwickelt hat und sogar die Luftfahrtbranche und die Landwirtschaft überrundet. Im Jahr 2002 spielten die Produktionen der grössten Hollywood-Gesellschaften im Ausland Einnahmen von rund 9,6 Milliarden Dollar ein, davon 60 Prozent allein in Europa. Laut Angaben der Europäischen audiovisuellen Informationsstelle lag der Anteil der amerikanischen Filme am europäischen Kinofilmmarkt 2002 bei 71,2 Prozent ${ }^{57}$. Die amerikanische Reaktion liess im Übrigen nicht auf sich warten: Der neue amerikanische Vertreter bei der UNESCO, Terrel Richard Miller, signalisierte bereits, dass sein Land Diskussionen über dieses Übereinkommen grundsätzlich ablehnen werde ${ }^{58}$.

Trotz dieser Polemik begrüsste der UNESCO-Direktor Koichiro Matsuura die Rückkehr der Vereinigten Staaten, die es der nunmehr 190 Mitglieder starken Organisation erlaube, einen entscheidenden Schritt hin zur Universalität zu

56 Informationsdienst des Aussenministeriums der Vereinigten Staaten, Les Etats-Unis et l'UNESCO, Website $<$ www.usinfo.state.gov/francais/>.

57 „Deux ans de travaux pour une convention“, Le Monde, 13. Oktober 2003.

58 Ibid. 
vollziehen. Ausserdem betonte er, dass der amerikanische Jahresbeitrag an die UNESCO von 53 Millionen Dollar eine höchst willkommene Aufstockung des Budgets ermögliche ${ }^{59}$. Das finanzielle Engagement der Vereinigten Staaten entspricht rund 22 Prozent des Gesamthaushalts.

\section{QUELLEN}

Nationale Schweizerische UNESCO-Kommission, Die Schweiz und die UNESCO. Fragen und Antworten, Bern, 1991.

Eidgenössisches Departement für auswärtige Angelegenheiten, Die Schweiz nimmt Einsitz in drei Unterorgane der UNESCO, Pressemitteilung, 14. Oktober 2003.

Aussenministerium der Vereinigten Staaten, Informationsdienst, Les Etats-Unis et l'UNESCO, $<$ www.usinfo.state.gov/francais/>.

UNESCO, Pressemitteilungen zur 32. Generalkonferenz der UNESCO.

UNESCO Info. Newsletter der Schweizerischen UNESCO-Kommission, monatliche Veröffentlichung, einsehbar auf der Website <www.unesco.ch>.

Crucifix Isabelle (Dir.), Organisations internationales à vocation universelle, Paris, La Documentation Française, 1993.

Le Monde, „De retour à l'UNESCO, Washington lance l'offensive contre le projet de convention sur la diversité culturelle“, 30. September 2003; „Deux ans de travaux pour une convention“, 13. Oktober 2003.

Le Temps, „Les Etats-Unis réintègrent l’UNESCO“, 30. September 2003; „David Streiff: ,La Suisse a beaucoup à apporter“", 15. Oktober 2003.

Neue Zürcher Zeitung, „Washingtons Rückkehr in die UNESCO“, 1. Oktober 2003.

St.Galler Tagblatt, „,Von bestürzender Bedeutungslosigkeit““, 1. Oktober 2003.

\section{INTERNET-ADRESSEN}

Internationales Jahr des Süsswassers (offizielle Website) : <www.wateryear2003.org >.

Schweizerische UNESCO-Kommission: <www.unesco.ch>.

Informationsdienst des Aussenministeriums der Vereinigten Staaten: <www.usinfo.state.gov/francais>.

UNESCO: <www.unesco.org >.

\subsection{Internationale Konferenzen}

\section{$\square$ Internationale Arbeitskonferenz}

Vom 3. bis 19. Juni 2003 tagte die 91. Arbeitskonferenz der Internationalen Arbeitsorganisation (IAO) ${ }^{60}$ in Genf. Die Teilnehmer und Teilnehmerinnen debattierten über Massnahmen zur Lösung sozialer und arbeitsspezifischer Fragen und Probleme. Sie verabschiedeten eine Konvention über die Identität von Seeleuten, starteten eine Kampagne zur Förderung eines erweiterten Sozialschutzes, diskutierten einen Bericht über Diskriminierung bei der Arbeit und beschlossen das Budget für die Jahre 2004-2005. Das Budget liegt mit 530 Millionen US-Dollar trotz Spardruck auf der Höhe der Vorjahre.

Einen Schwerpunkt der Konferenz bildete die Verabschiedung einer globalen Strategie im Bereich Sicherheit und Gesundheit am Arbeitsplatz. Eine Kultur der Prävention und spezifische Instrumente sollen die Zahl der Berufsunfälle und -krankheiten senken.

59 „Les Etats-Unis réintègrent l'UNESCO“, Le Temps, 30. September 2003; „Washingtons Rückkehr in die UNESCO“, Neue Zürcher Zeitung, 1. Oktober 2003; „,Von bestürzender Bedeutungslosigkeit“, St.Galler Tagblatt, 1. Oktober 2003.

60 International Labour Conference 91st Session, 3-19 June 2003, <www.ilo.org/public/english/standards/ relm/ilc/ilc91>. 
Die Kommission für die Umsetzung der Normen analysierte die Lage der Arbeitnehmer und Arbeitnehmerinnen in 25 Ländern. Sie kritisierte namentlich die fehlende Gewerkschaftsfreiheit in Weissrussland und in Burma und forderte die Regierungen zur Respektierung der diesbezüglichen Konvention Nr. 87 auf. Ebenso besorgt zeigte sich die Kommission über die Arbeitssituation in Kolumbien, Kamerun, Libyen, Mauretanien und Simbabwe.

Anlässlich der Konferenz kritisierte der Schweizer Delegierte der Arbeitnehmer, Jean-Claude Prince, die prekären Arbeitsbedingungen bei den Vereinten Nationen. Die UNO verweigere ihren Angestellten Arbeitnehmerrechte, welche in ihrem eigenen Schoss ausgearbeitet werden, so der Delegierte. Von den 23'000 Mitarbeitern des UNO-Systems in Genf verfügen nahezu die Hälfte lediglich über befristete und wenig Schutz bietende Arbeitsverträge ${ }^{61}$.

Die Schweiz und die IAO haben im Juni 2003 die Fortsetzung eines Programms zur Förderung des Dialogs zwischen Sozialpartnern und Regierung, des Aufbaus friedlicher Streitbeilegungsmechanismen sowie der Einhaltung von Sozialstandards in Unternehmen im südlichen Afrika unterzeichnet. Das von der Schweiz mit 2,5 Millionen US-Dollar finanzierte Programm wurde auf die Staaten Angola und Mosambik ausgeweitet und umfasst nun das ganze südliche Afrika ${ }^{62}$.

\section{$\square$ Weltgesundheitsversammlung der WHO}

Die 56. Weltgesundheitsversammlung fand vom 19. bis 28. Mai 2003 in Genf statt. Im Zentrum der Versammlung standen die Lungenkrankheit SARS, die Rahmenkonvention gegen den Tabakmissbrauch, die HIV/Aids-Epidemie und die Ernennung des Koreaners Jong Wook Lee zum Generaldirektor der Weltgesundheitsorganisation (WHO). Ausserdem wurde das reguläre Budget für die Jahre 2004-2005 verabschiedet. Dieses wurde um knapp 3 Prozent erhöht und beträgt nun jährlich 440 Millionen US-Dollar ${ }^{63}$.

Am 12. März 2003 hatte die WHO wegen einer mysteriösen Lungenkrankheit zum ersten Mal in ihrer Geschichte weltweit Alarm geschlagen. Daher stand die Infektionskrankheit SARS im Zentrum der Konferenz und führte zur Verabschiedung zweier Resolutionen. Die erste betraf die Revision des internationalen Sanitätsreglements, welches die internationale Zusammenarbeit im Bereich der Kontrolle von wichtigen Infektionskrankheiten regelt. Die zweite Resolution betraf direkt die neue Lungenkrankheit. Diese wurde als erste schlimme Infektionskrankheit des 21. Jahrhunderts bezeichnet. Die Länder wurden aufgefordert, den Kampf gegen SARS voll zu unterstützen und die weltweite Zusammenarbeit und Vernetzung zur Bekämpfung der Infektionskrankheiten zu intensivieren ${ }^{64}$.

61 Pressemitteilung SGB, „Die internationalen Arbeitsnormen müssen auch für die UNO selbst gelten“, Bern, Juni 2003.

62 Pressemitteilung Bund, „Förderung des Dialogs zwischen Sozialpartnern im südlichen Afrika“, Bern, Juni 2003.

63 Dazu kommen noch 700 Millionen US-Dollar pro Jahr an extrabudgetären projektbezogenen Beiträgen.

64 Die rasche Eindämmung von SARS kann von der WHO und der Wissenschaft als Erfolg verbucht werden. Zwei Wochen nach dem WHO-Alarm war das SARS-Virus identifiziert und weitere zwei Wochen später sein Erbgut entschlüsselt. Die Eindämmungsstrategie der WHO hatte jedoch negative wirtschaftliche Konsequenzen. Neue Zürcher Zeitung, „SARS - eine Seuche als Erfolgsgeschichte“, 7. Juli 2003. 
Einstimmig verabschiedeten die 192 Staaten der Weltgesundheitsorganisation die in mehreren Jahren erarbeitete Rahmenkonvention über die Tabakkontrolle. Die Anti-Tabak-Konvention stellte eine der Prioritäten der scheidenden Generaldirektorin Gro Harlem Brundtland dar und ist der erste rechtlich verbindliche Vertrag in der Geschichte der WHO. Die Konvention verbietet die Werbung für Tabakprodukte in den Mitgliedsstaaten. Zudem sieht die Vereinbarung höherer Zigarettenpreise, schärfere Massnahmen gegen den Zigarettenschmuggel und einen besseren Schutz der Nichtraucher und Nichtraucherinnen vor dem Passivrauchen vor. Die Konvention tritt in Kraft, sobald sie von mindestens 40 Staaten ratifiziert worden ist ${ }^{65}$.

Nach Schätzung der WHO sterben derzeit jährlich 5 Millionen Menschen an den Folgen des Tabakkonsums. Die Zahl der Todesopfer könnte ohne konkrete Gegenmassnahmen drastisch ansteigen und vor allem die Entwicklungsländer hart treffen, befürchtet die WHO. Während die Zahl der Raucher und Raucherinnen in den Industrieländern zurückgeht, werden in den südlichen Ländern immer mehr Zigaretten konsumiert. Diese Staaten waren es denn auch, die sich am vehementesten für ein totales Werbeverbot für Tabakprodukte eingesetzt hatten.

Die Schweiz zeigte sich zufrieden über die Verabschiedung der Rahmenkonvention über die Tabakkontrolle. Gemäss dem Direktor des Bundesamtes für Gesundheit, Thomas Zeltner, wird die Schweiz das Abkommen aber kaum vor dem Jahr 2006 ratifizieren. Sie liege in diesem Bereich der Gesundheitspolitik verglichen mit anderen Ländern deutlich im Hintertreffen und müsse vor einer Ratifizierung noch Gesetzesänderungen vornehmen. Einzelne Punkte der Konvention können aber auf dem Verordnungsweg rasch umgesetzt werden ${ }^{66}$.

Der Bundesrat will der Weltgesundheitsorganisation und dem HIV/Aids-Programm der Vereinten Nationen (UNAIDS) zum Bau eines neuen Verwaltungsgebäudes in Genf ein zinsfreies Darlehen über 60 Millionen Franken zur Verfügung stellen. Dazu legte er dem Parlament im April 2003 eine entsprechende Botschaft vor ${ }^{67}$.

\section{QUELLEN}

Neue Zürcher Zeitung, „,Diskriminierung am Arbeitsplatz“, 12. Mai 2003.

\section{INTERNET-ADRESSEN}

Informationskampagne des Bundesamtes für Gesundheit zu den Risiken des Tabakkonsums: <www. rauchenschadet.ch>.

Internationale Arbeitsorganisation (IAO) : <www.ilo.org $>$.

Weltgesundheitsorganisation (WHO) : <www.who.int $>$.

65 Stand Dezember 2003: 80 Unterschriften, 5 Ratifikationen.

66 Neue Zürcher Zeitung, „Schweiz sehr zufrieden über Anti-Tabak-Konvention“, 21. Mai 2003, und Schweizerische Stiftung für rauchfreie Luft und gegen die Tabaksucht, Pro Aere Info, Ausgabe 2/03.

67 Bundesrat, Botschaft über die Gewährung eines Darlehens an die Immobilienstiftung für die internationalen Organisationen (FIPOI) zur Finanzierung eines neuen Gebäudes für WHO und UNAIDS in Genf (03.031), Bern, 16. April 2003 (BB1 2003 3439). 
Im Frühjahr 2003 nahm der von der Schweiz unterstützte Internationale Strafgerichtshof im niederländischen Den Haag seine Arbeit auf. Dies bedeutet nach dem In-Kraft-Treten des Römer Statutes im Jahr 2002 einen weiteren Schritt in Richtung einer internationalen Strafverfolgung schwerwiegender Verbrechen und Menschenrechtsverletzungen.

Das von der UNO-Generalversammlung Ende 2002 verabschiedete Fakultativprotokoll zur Antifolterkonvention stellte für die Schweiz einen aussenpolitischen Erfolg dar, hatte sie sich doch seit vielen Jahren für ein internationales System zur Folterprävention stark gemacht.

Auf schweizerischer Ebene wurde der Bundesrat vom Nationalrat verpflichtet, eine eidgenössische Menschenrechtskommission zu bilden. Der Bundesrat seinerseits nahm mit dem Iran einen Menschenrechtsdialog auf. Die Schweiz erhofft sich damit mittelfristig eine signifikante Verbesserung der iranischen Menschenrechtslage.

\section{$\square$ Internationaler Strafgerichtshof}

Der Internationale Strafgerichtshof (IStGH) basiert auf dem Römer Statut von 1998, das von 139 Staaten unterzeichnet wurde. Bis Oktober 2003 hatten 92 Länder das Statut ratifiziert und sind damit zu Vertragsstaaten geworden. Die von der UNO unabhängige Institution ist für Verfahren wegen Völkermord, Verbrechen gegen die Menschlichkeit und Kriegsverbrechen zuständig, sofern der betroffene Staat nicht willens oder nicht in der Lage ist, die Ermittlung und Strafverfolgung selbst durchzuführen. Zudem muss der Staat, in dem die Tat begangen wurde beziehungsweise aus dem der Täter oder die Täterin stammt, Vertragspartei sein.

Gleichzeitig mit der Vereidigung der ersten 18 Richter und Richterinnen am 11. März 2003 nahm der Internationale Strafgerichtshof in Den Haag seine Arbeit auf ${ }^{68}$. An der konstituierenden Sitzung des Gerichts wurde ausserdem der Kanadier Philippe Kirsch von seinen Richterkollegen und -kolleginnen zum Präsidenten des IStGH gewählt ${ }^{69}$. Einen Monat später wählten die Vertragsparteien an ihrer zweiten Konferenz den Argentinier Luis MorenoOcampo einstimmig zum Chefankläger. Richter, Richterinnen und Chefankläger üben ihr Mandat für eine Dauer von neun Jahren aus.

An seiner ersten Pressekonferenz vom 16. Juli 2003 informierte Chefankläger Moreno-Ocampo über die Klagen an den IStGH und über die zukünftigen Schwerpunkte des Gerichtes ${ }^{70}$.

Im ersten Jahr seines Bestehens hat das Gericht 499 Eingaben aus 66 Ländern erhalten. Eine grosse Zahl der eingegangenen Klagen betrafen den Irak, der allerdings das Römer Statut nicht ratifiziert hat und daher nicht unter die Jurisdiktion des Internationalen Strafgerichts fällt.

68 Pressemitteilung Internationaler Strafgerichtshof, 11. März 2003, <www.icc-cpi.org>.

69 Die von der Schweiz zur Richterin vorgeschlagene Barbara Ott wurde nicht gewählt. Der Präsident des IStGH, Philippe Kirsch, wählte den Schweizer Völkerrechtsexperten Valentin Zellweger zu seinem persönlichen Berater und zum Stabschef des Gerichtspräsidiums.

70 Pressemitteilung Internationaler Strafgerichtshof, 16. Juli 2003, <www.icc-cpi.org >. 
Es gingen ebenfalls Klagen über Menschenrechtsverletzungen in der Demokratischen Republik Kongo ein. Die Lage im Kongo wurde vom Büro des Chefanklägers als äusserst schwer beurteilt ${ }^{71}$. Daher möchte der Strafgerichtshof vorrangig in diesem Staat und speziell in der Region Ituri ermitteln. Vor diesem Hintergrund forderte der Chefankläger die Vertragsstaaten auf, eigene Ermittlungen über finanzielle Beziehungen von Personen und Firmen in der Demokratischen Republik Kongo durchzuführen. Dabei geht es um die Beteiligung am illegalen Handel mit Diamanten, Gold, Koltan und anderen Rohstoffen aus dem zentralafrikanischen Land. Mit dem Erlös aus diesen Geschäften würden grausame Machtkämpfe finanziert, unter denen die kongolesische Zivilbevölkerung schwer zu leiden habe. So stünden Verbrechen gegen die Menschenrechte, die sich in der Region Ituri ereigneten, in direktem Zusammenhang mit dem Kampf um die Kontrolle über die Rohstoffgewinnung, sagte der Chefankläger.

Die USA setzten auch 2003 ihren Obstruktionskurs gegen den Internationalen Strafgerichtshof fort. Auf Druck der USA verlängerte der UNO-Sicherheitsrat im Juni 2003 die Immunität von in UNO-Missionen beteiligten US-Bürgern und Bürgerinnen um ein Jahr ${ }^{72}$. 12 der 15 Mitglieder des Gremiums stimmten für die entsprechende Resolution; Deutschland, Frankreich und Syrien enthielten sich der Stimme. Die Schweiz hatte sich in einer offenen Debatte des Sicherheitsrates gegen die Forderungen nach Immunität ausgesprochen. Es sei besorgniserregend, wenn der Sicherheitsrat die Bedeutung eines multilateralen Abkommens (das Römer Statut) zu reduzieren versuche. Die Resolution stelle die internationale Strafgerichtsbarkeit und die Friedensmissionen in einen Widerspruch. Dabei seien die beiden Instrumente komplementär, so Jenö Staehelin, UNOBotschafter der Schweiz ${ }^{73}$.

Den USA ist es ausserdem mit viel Druck gelungen, Mitgliedsstaaten des Gerichts zu bilateralen Abkommen zu bewegen. Bis Oktober 2003 hatten über 60 Staaten solche Abkommen unterzeichnet oder bereits ratifiziert. Die Abkommen sollen verhindern, dass der entsprechende Staat amerikanische Staatsangehörige an den Internationalen Strafgerichtshof ausliefert. Länder welche kein solches Abkommen unterzeichnen, müssen mit dem Abbruch der Militärhilfe rechnen ${ }^{74}$. Fürs Fiskaljahr 2003 strichen die USA die Militärhilfe für 32 verbündete Staaten, darunter die Slowakei, Bulgarien sowie die baltischen Länder.

Jahrbuch 2003, S. 160-162; 2002, S. 243-255.

Im November 2003 stellte die Schweizerische Gesellschaft gegen Straflosigkeit TRIAL (Track Impunity Always) ihr Handbuch „Der Kampf gegen Straflosigkeit im schweizerischen Recht" vor $^{75}$. Die Publikation definiert die Tatbestände des

71 Laut Schätzungen sind im bisherigen Kriegsverlauf zwischen 2,5 und 3,3 Millionen Menschen ums Leben gekommen.

72 Die neue Resolution 1487 des UNO-Sicherheitsrates ersetzt die entsprechende Resolution von 2002 (Nr. 1422).

73 Staehelin Jenö, Statement on the Meeting of the Security Council on the Proposed renewal of the provisions of Security Council resolution 1422, New York, 12. Juni 2003.

74 Coalition for the International Criminal Court, „US and the ICC“, <www.iccnow.org >.

75 Die im Jahr 2002 von Juristen, Folteropfern und Verfechtern der Menschenrechte gegründete TRIAL hat zum Ziel, sich im Kampf gegen die Straflosigkeit von Völkermördern, Folterern und Kriegsverbrechern zu engagieren. Mit Hilfe eines gesamtschweizerischen Anwaltsnetzes sollen die Opfer internationaler Verbrechen bei der Einreichung von Klagen unterstützt werden, sofern sich die Täter in der Schweiz aufhalten. 
Genozids, der Kriegsverbrechen, der Verbrechen gegen die Menschlichkeit, sowie der Folter und thematisiert die Aspekte Verjährung, Immunität und Amnestie. Es richtet sich an Anwälte und Anwältinnen, Nichtregierungsorganisationen und Opfer von internationalen Verbrechen und soll ihnen als Hilfsmittel für die Verfolgung von Tätern in der Schweiz dienen ${ }^{76}$.

\section{$\square$ Session der UNO-Menschenrechtskommission}

In Genf fand vom 15. März bis 26. April die 59. Session der UNO-Menschenrechtskommission statt. Während der sechswöchigen Arbeiten verabschiedete die Kommission 86 Resolutionen, 18 Beschlüsse und 3 Deklarationen zu den Themen wirtschaftliche, soziale und kulturelle Rechte, bürgerliche und politische Rechte sowie Kinderrechte. Zum ersten Mal wurden Resolutionen zur Menschenrechtssituation in den Ländern Nordkorea, Turkmenistan und Weissrussland beschlossen. Resolutionsentwürfe über die Menschenrechtslage in Russland (Tschetschenien) und im Sudan wurden dagegen abgelehnt. Die Kommission nominierte vier neue Sonderberichterstatter zu den Themen: „Prävention von durch Kleinwaffen verursachte Menschenrechtsverletzungen“, „Rückgabe von Wohnraum und Besitz bei der Rückkehr von Flüchtlingen und intern Vertriebenen“, „Souveränität autochthoner Völker über natürliche Ressourcen“ und „Diskriminierung in Strafgerichten“.

Vor dem Hintergrund des Irakkrieges wurde ein Antrag für eine ausserordentliche Sitzung über die Menschenrechte und die humanitäre Situation im Irak gestellt. Der Antrag wurde jedoch mit 25 gegen 17 Stimmen abgelehnt. Am letzten Sitzungstag verabschiedete die Kommission dennoch eine Resolution über die Menschenrechte im Irak, welche die Menschenrechtsverletzungen der irakischen Regierung verurteilt. Die Kriegsparteien werden zur Respektierung des humanitären Völkerrechts aufgefordert und die internationale Gemeinschaft wird gebeten, humanitäre Hilfe zu leisten und den Irak bei der Bildung von demokratischen und rechtsstaatlichen Institutionen zu unterstützen.

Die Arbeiten der Kommission waren oftmals von politischen Kämpfen und Absprachen zwischen Ländern oder Ländergruppen geprägt, was der Sache der Menschenrechte wenig zuträglich war. Dies veranlasste verschiedene NRO, aber auch den UNO-Hochkommissar für Menschenrechte, Sergio Viera de Mello, sowie UNO-Generalsekretär Kofi Annan, die Kommission zurechtzuweisen und Reformen zu fordern. Über mögliche Reformen - namentlich die Änderung der Zusammensetzung der Menschenrechtskommission - sprach auch Bundesrätin Calmy-Rey in ihrer Rede an die Kommission: „Wäre es nicht sinnvoller, die Mitgliedschaft in der Kommission auf jene [Länder] zu beschränken, die bereit sind, diese Instrumente [die sechs grundlegenden universellen Menschenrechtsübereinkommen und deren Überwachungsmechanismen] anzuwenden, die Mechanismen der Kommission zu nutzen und mit ihr zusammenzuarbeiten ?"77.

Die Schweiz beschloss, ihren finanziellen Beitrag ans UNO-Hochkommissariat für Menschenrechte auf 1,5 Millionen Franken zu verdreifachen ${ }^{78}$. Doch nicht

76 Pressemitteilung, TRIAL „Die Schweiz darf kein sicherer Hafen für Kriegsverbrecher werden“, Bern, 3. November 2003, <www.trial-ch.org >.

77 59. Sitzung der UNO-Menschenrechtskommission, Rede von Bundesrätin Micheline Calmy-Rey, Genf, 18. März 2003.

78 Die Schweiz gehört damit zu den 10 wichtigsten Beitragszahlern; 24 heures, „,Droits de 1'homme: la Suisse triple son soutien financier à l’ONU“, 11. März 2003. 
nur finanziell möchte die Schweiz eine grössere Rolle spielen; sie strebt bis zum Jahr 2007 einen Sitz in der UNO-Menschenrechtskommission an.

\section{$\square$ Internationale Konventionen}

\section{Verabschiedung des Fakultativprotokolls zur Folterkonvention}

Ende 2002 verabschiedete die UNO-Generalversammlung das Fakultativprotokoll zum Übereinkommen gegen Folter und andere grausame, unmenschliche oder erniedrigende Behandlung oder Strafe. 127 Staaten stimmten für das Protokoll, 42 enthielten sich der Stimme und vier Staaten (USA, Nigeria, MarshallInseln, Palau) stimmten dagegen. Durch das Fakultativprotokoll wird erstmals auf universeller Ebene ein Instrument zur Folterprävention geschaffen mit dem Ziel, die Vertragsstaaten der Folterkonvention ${ }^{79}$ bei der Erfüllung ihrer Verpflichtungen zu unterstützen.

Der vorgesehene präventive Mechanismus beruht auf einem internationalen und einem nationalen Pfeiler. Kernstück beider Pfeiler bildet ein System regelmässiger Besuche unabhängiger Gremien von Gefängnissen, Polizeistationen und anderen Orten, in denen Menschen inhaftiert sind (duales Besuchssystem) ${ }^{80}$. Diese Aufgabe wird auf internationaler Ebene einem neu zu bildenden Subkomitee zugewiesen, während auf nationaler Ebene unabhängige Gremien geschaffen werden. Die Vertragsstaaten sind verpflichtet, dem Subkomitee und den nationalen Gremien freien Zugang zu den Orten und Personen ihrer Wahl wie auch zu den relevanten Informationen zu gewähren. Ein Fonds soll Mittel bereitstellen, um die Empfehlungen des Subkomitees umzusetzen und die nationalen Gremien auszubilden.

Das Ziel eines internationalen Systems zur Folterprävention wurde seit den 70er Jahren vom Genfer Jean-Jaques Gautier und seiner Organisation Comité Suisse contre la torture verfolgt ${ }^{81}$. Später nahm die Schweiz das Projekt auf und verfolgte es weiter. Im Jahr 1990 brachte Costa Rica das Projekt für die Schweiz in die UNO ein, und zwei Jahre danach nahm eine Arbeitsgruppe die Arbeit an einem Fakultativprotokoll zur Folterkonvention auf. Es gelang jedoch erst im Jahr 2002, dank des Konzepts des dualen Besuchssystems, einen mehrheitsfähigen Kompromiss zu finden. Die Verabschiedung des Fakultativprotokolls kann als Erfolg der schweizerischen Aussenpolitik gewertet werden. Es tritt in Kraft, sobald es von 20 Staaten ratifiziert ist ${ }^{82}$.

\section{In-Kraft-Treten des Protokolls Nr. 13 der Europäischen Menschenrechtskonvention}

Mit dem im Juli 2003 in Kraft getretenen Protokoll Nr. 13 machte der Europarat den letzten Schritt zur vollständigen Abschaffung der Todesstrafe ${ }^{83}$. Das Protokoll verpflichtet die Vertragsstaaten, auch in Kriegszeiten oder bei unmittelbarer Kriegsgefahr niemanden zur Todesstrafe zu verurteilen oder hinzurichten. Bis

79 Das Übereinkommen gegen Folter und andere grausame, unmenschliche oder erniedrigende Behandlung oder Strafe wurde 1984 verabschiedet und bis 2003 von 133 Staaten ratifiziert.

80 Die Mitgliedsstaaten des Europarates kennen mit dem Europäischen Abkommen zur Verhütung von Folter und unmenschlicher oder erniedrigender Behandlung oder Strafe bereits seit 1989 ein solches Besuchssystem.

81 Die Organisation heisst heute Association pour la Prévention de la Torture, <www.apt.ch >.

82 Stand Oktober 2003: 21 Unterschriften, 2 Ratifikationen.

83 Europarat, ,Human Rights“, <www.ceo.int/T/E/Human_rights $>$. 
Oktober 2003 hatten 19 Staaten das Protokoll ratifiziert, darunter die Schweiz. Von den 45 Mitgliedsstaaten des Europarates haben Armenien, Aserbaidschan, Russland und die Türkei das Protokoll weder ratifiziert, noch unterzeichnet.

\section{In-Kraft-Treten der Wanderarbeiter-Konvention}

Im Juli 2003 ist die von der UNO-Generalversammlung im Jahr 1990 verabschiedete Wanderarbeitnehmer-Konvention in Kraft getreten ${ }^{84}$. Diese jüngste umfassende Menschenrechtskonvention formuliert, welche Rechte den Arbeitsmigranten, -migrantinnen und ihren Familien zustehen ${ }^{85}$.

Als Kontrollinstrument sieht die Konvention einen Ausschuss vor, dem die Staaten ein Jahr nach In-Kraft-Treten der Konvention einen Erstbericht und danach alle fünf Jahre Folgeberichte zur Prüfung vorlegen. Der Ausschuss kann auch zur Entgegennahme und Prüfung von Individualbeschwerden ermächtigt werden.

Die Schweiz will die Unterzeichnung der Konvention zurzeit nicht prüfen. Sie halte es für problematisch, illegal anwesenden Migranten Rechte einzuräumen, welche über die allgemeinen Menschenrechte hinausgehen ${ }^{86}$.

[D] Jahrbuch 2004, Nr. 1, in Kap. 12.1., „Aussenpolitische Massnahmen im Bereich der Migration“. S. 223-224.

\section{$\square$ Menschenrechtsdialog der Schweiz}

Der Menschenrechtsdialog ist ein Instrument der schweizerischen Menschenrechtspolitik. Mit seiner Hilfe sollen Beeinträchtigungen der Menschenrechte bekämpft, deren Einhaltung gefördert und die Schaffung der zu diesem Zweck erforderlichen Rahmenbedingungen (Rechtsstaat, Demokratie) unterstützt werden $^{87}$. In den 90er Jahren führte das EDA mit fünf Staaten einen offiziellen Menschenrechtsdialog: China (Beginn 1992), Marokko, Vietnam, Pakistan (Beginn 1997) und Kuba (Beginn 1999). Im Herbst 2000 wurde aufgrund einer externen Evaluation entschieden, die Bemühungen auf den Dialog mit China zu konzentrieren und die Gespräche mit den anderen Ländern zu beenden ${ }^{88}$.

Es ist umstritten, ob der Menschenrechtsdialog mit China positive Veränderungen der chinesischen Menschenrechtslage bewirkt ${ }^{89}$. Laut Bundesrat konnte sich das EDA aber in den letzten Jahren dank des Dialogs mit China hinsichtlich vieler konzeptioneller Fragen Klarheit verschaffen. Die gewonnenen Erkenntnisse sollen im Rahmen von neuen Dialogen genutzt werden. Folgende Kriterien sind für die Auswahl von Dialogpartnerstaaten besonders ausschlaggebend:

84 Bis Juli 2003 wurde die Konvention von 22 Staaten ratifiziert; darunter befindet sich kein westliches Einwanderungsland.

85 Information Kit on the United Nations Convention on Migrants Rights: <www.unesco.org/most/ migration/convention>.

86 Schweizerische Depeschenagentur (sda), 10. September 2003.

87 Bundesrat, Botschaft über einen Rahmenkredit für Massnahmen zur zivilen Konfliktbearbeitung und Menschenrechtsförderung (02.076), S. 8034-8036, Bern, 23. Oktober 2003 (BB1 2002 7975).

88 Die Evaluation des Instituts für öffentliches Recht der Universität Bern war zum Schluss gekommen, dass es der Politik der Menschenrechtsdialoge an Klarheit in der inhaltlich-konzeptionellen Stossrichtung, der geografischen Ausrichtung, der verfolgten Zielrichtung sowie an personellen Kapazitäten mangle (ibd.).

89 Sutter Ruedi, „Tibet, die Schweiz und der Menschenrechtsdialog mit China“, 29. November 2001, $<$ www.onlinereports.ch/ChinaTibet.htm> und Neue Zürcher Zeitung, „Schweizer Menschenrechtsdelegation in China“, 28. Januar 2002. 
- Im entsprechenden Land herrscht eine prekäre Menschenrechtslage.

- Die Regierung im betreffenden Staat ist an einem offenen und kritischen Dia$\log$ interessiert und zeigt einen echten Willen zur Veränderung.

- Zwischen der Schweiz und dem betreffenden Staat bestehen substanzielle bilaterale Beziehungen oder ungenutzte Potenziale für solche Beziehungen.

Diese Kriterien scheinen nun auch auf den Iran zuzutreffen - einen Staat, mit welchem die Schweiz ,gute enge und vielseitige Beziehungen“"90 pflegt. Der Vorschlag von Bundesrat Joseph Deiss während seines Besuchs im Iran (Oktober 2002), einen Menschenrechtsdialog zwischen den beiden Ländern aufzunehmen, wurde von den iranischen Behörden positiv beantwortet. Die erste Gesprächsrunde fand im Oktober 2003 in Teheran statt ${ }^{91}$. Schwerpunkte der Gespräche waren die Abschaffung von Körperstrafen, die Verbesserung der Situation in den Gefängnissen sowie die Ratifikation internationaler Menschenrechtsinstrumente. Der Iran und die Schweiz wollen im Bereich des Strafvollzugs künftig enger zusammenarbeiten. Gemäss Simon Amman, Programmverantwortlicher für den Mittleren Osten in der Sektion Menschenrechtspolitik des EDA, ist die Menschenrechtsarbeit im Iran ein langfristiges Projekt. Regelmässige, offene und transparente Diskussionen, verbunden mit der Unterstützung der Zivilgesellschaft, dürften die Menschenrechtslage bedeutend verbessern. Wichtig sei dabei, dass der Iran als Partnerland betrachtet und mit dem nötigen Respekt behandelt werde ${ }^{92}$.

\section{$\square$ Eidgenössische Kommission für Menschenrechte}

Im Jahr 2003 wurden weitere Schritte in Richtung einer eidgenössischen Menschenrechtsinstitution getan. Nachdem der Ständerat bereits im Oktober 2002 den Bundesrat beauftragt hatte, einen Bericht über die mögliche Gründung einer schweizerischen Menschenrechtskommission zu erstellen, sprach sich auch Bundesrätin Micheline Calmy-Rey an der Delegiertenversammlung von Amnesty International für die Schaffung einer nationalen Kommission für Menschenrechte aus ${ }^{93}$.

Am 20. Juli 2003 ging der Nationalrat einen Schritt weiter als der Ständerat und befürwortete die parlamentarischen Initiative zur Einrichtung einer Menschenrechtsinstitution ${ }^{94}$. Um den Weisungen des Parlaments Folge zu leisten, wurde im Auftrag des EDA ein Grundlagenbericht ausgearbeitet. Dieser präsentiert sechs mögliche Modelle, welche untereinander kombinierbar sind ${ }^{95}$. Ende Okto-

90 Scheurer Guillaume, „Etat actuel des relations bilatérales vues de l'Ambassade suisse à Téhéran“, in Politorbis, Nr. 33, Zentrum für Analyse und prospektive Studien, Bern, 2003.

91 Pressemitteilung EDA, 16. Oktober 2003.

92 Amman Simon, „Verhandlung statt Verurteilung: Die Schweiz beginnt in diesem Jahr den Menschenrechtsdialog mit Iran“, in Politorbis, Nr. 33, Zentrum für Analyse und prospektive Studien, Bern, 2003.

93 Swissinfo, „Calmy-Rey will Kommission für Menschenrechte“, 14. April 2003.

94 Parlamentarische Initiative, „Eidgenössische Kommission für Menschenrechte“ (01.461), eingereicht von Vreni Müller-Hemmi.

95 Folgende Modelle wurden vorgeschlagen: Explizite Menschenrechtsmandate an bestehende Institutionen, Neue beratende Menschenrechtskommission, Kommissionsdach Plus, Comité des sages, Unabhängiges Fachinstitut, Ombudsstelle. Erika Schläppi, Möglichkeiten für die Schaffung einer nationalen Menschenrechtsinstitution: Kurzfassung der Ergebnisse einer Grundlagenstudie: <www. humanrights.ch/schweiz/eidg_kommission>. 
ber 2003 trafen sich rund 40 Nichtregierungsorganisationen, die eigentlichen Initianten des Projekts, zu einer Arbeitstagung und diskutierten Ausgestaltung, Funktionen und Kompetenzen einer zukünftigen schweizerischen Menschenrechtsinstitution ${ }^{96}$. Die NRO einigten sich nicht auf ein institutionelles Modell. Ihnen ist vor allem eine klare gesetzliche Grundlage und eine grosse politische sowie finanzielle Unabhängigkeit wichtig. Die Institution soll in erster Linie Menschenrechtsverletzungen in der Schweiz beobachten sowie die Behörden und die breite Bevölkerung sensibilisieren. Bezüglich der schweizerischen Aussenpolitik forderten die NRO eine Überwachung und Förderung der Kohärenz im Menschenrechtsbereich.

Die Ergebnisse der Tagung sollen als Stellungnahme der NRO in den Bericht des Bundesrates einfliessen ${ }^{97}$.

\section{QUELLEN}

Bundesrat, Botschaft über einen Rahmenkredit für Massnahmen zur zivilen Konfliktbearbeitung und Menschenrechtsförderung (02.076), Bern, 23. Oktober 2003 (BB1 2002 7975).

EDA, ABC des humanitären Völkerrechts, Bern, April 2003.

Humanrights.ch, „Folter à discrétion ?“"Menschenrechte Schweiz MERS, April 2003.

Kompetenzzentrum Friedensförderung, KOFF Newsletter 09/2003, „Wie sehen NRO eine schweizerische Menschenrechtsinstitution?“, „Gespräche mit dem Iran zu Menschenrechtsfragen“, Bern, 1. November 2003.

Le Temps, „Malgré l'hostilité américaine, la Cour pénale internationale entre en fonction à La Haye“, 11. März 2003.

Moniteur de la Cour Pénale Internationale, Nr. 23 (Februar 2003); Nr. 24 (April 2003); Nr. 25 (September 2003).

Pressemitteilung Bundesrat, Zweite Versammlung der Vertragsstaaten des Internationalen Strafgerichtshofs, Bern, 27. August 2003.

Zentrum für Analyse und prospektive Studien (EDA), Politorbis, Nr. 33, „Iran, Wirklichkeiten in Bewegung“, Bern, 2003

\section{INTERNET-ADRESSEN}

Association for the Prevention of Torture: <www.apt.ch>.

Association Internet pour la défense et la promotion des droits de l'homme (Aidh) : <www.aidh.org $>$ und $<$ www.droitshumains.org $>$.

Coalition for the International Criminal Court: $<w w w . i c c n o w . o r g>$.

December 18 (Online organization to promote and protect the rights of migrants): <www.december18. net>.

Europarat: <www.coe.int $>$.

Human Rights Watch: <www.hrw.org $>$.

International Service for Human Rights : <www.ishr.ch>.

Internationaler Strafgerichtshof (IStGH) : <www.icc-cpi.int $>$.

Menschenrechte Schweiz MERS : <www.humanrights.ch>.

Office of the High Commissioner of Human Rights : <www.unhchr.ch $>$.

59th session of the Commission on Human Rights : <www.unhchr.ch/html/menu2/2/59chr $>$.

96 Menschenrechte Schweiz, Eidgenössische Kommission für Menschenrechte - Übersicht, <www. humanrights.ch/schweiz/eidg_kommission>.

97 Der Bericht des Bundesrates über die Schaffung einer eidgenössischen Menschenrechtsinstitution soll im Frühjahr 2004 erscheinen. 